\title{
Observation of Top Quark Production in Proton-Nucleus Collisions
}

\author{
A. M. Sirunyan et al. \\ (CMS Collaboration) \\ (Received 21 September 2017; published 14 December 2017)
}

\begin{abstract}
The first observation of top quark production in proton-nucleus collisions is reported using proton-lead data collected by the CMS experiment at the CERN LHC at a nucleon-nucleon center-of-mass energy of $\sqrt{s_{N N}}=8.16 \mathrm{TeV}$. The measurement is performed using events with exactly one isolated electron or muon candidate and at least four jets. The data sample corresponds to an integrated luminosity of $174 \mathrm{nb}^{-1}$. The significance of the $t \bar{t}$ signal against the background-only hypothesis is above 5 standard deviations. The measured cross section is $\sigma_{t \bar{t}}=45 \pm 8 \mathrm{nb}$, consistent with predictions from perturbative quantum chromodynamics.
\end{abstract}

DOI: 10.1103/PhysRevLett.119.242001

The top quark, the heaviest elementary particle in the standard model, has been the subject of numerous detailed studies based on data samples with large integrated luminosities in $p \bar{p}$ and $p p$ collisions [1] accumulated at the Fermilab Tevatron and the CERN LHC, respectively. Until recently, top quark studies remained inaccessible in nuclear collisions because of the small integrated luminosities of the first heavy ion runs at the LHC and the low nucleon-nucleon $(N N)$ center-of-mass energies $\left(\sqrt{s_{N N}}\right)$ available at the BNL RHIC. This situation changed when the $2016 \mathrm{LHC}$ proton-lead $(p \mathrm{~Pb})$ run at $\sqrt{s_{N N}}=8.16 \mathrm{TeV}$ produced a data set corresponding to an integrated luminosity of $174 \mathrm{nb}^{-1}$ (equivalent to $36 \mathrm{pb}^{-1}$ of nucleonnucleon collision data). Top quark cross sections at the LHC are dominated by pair production via gluon-gluon fusion processes $(g g \rightarrow t \bar{t}+X)$, and are computable with great accuracy in perturbative quantum chromodynamics (QCD) $[2,3]$. In proton-nucleus collisions, the top quark is a novel and theoretically precise probe of the nuclear gluon density at high virtualities $Q^{2} \approx m_{t}^{2}$ (where $m_{t}$ is the top quark mass) in the unexplored high Bjorken- $x$ region $\left(x \gtrsim 2 m_{t} / \sqrt{s_{N N}} \approx 0.05\right)[4,5]$. In this region, "antishadowing" and "EMC" effects [6] are expected to modify the gluon density with respect to that in the free-proton case $[7,8]$. The production of top quarks thus provides information on the nuclear parton distribution functions (nPDF) that is complementary to that obtained through studies of electroweak boson production. In comparison to the $W$ and $Z$ cases [9,10], top-pair cross sections are more sensitive to gluon (rather than quark) densities at Bjorken- $x$ values about twice as large. Novel studies of parton energy loss

*Full author list given at the end of the article.

Published by the American Physical Society under the terms of the Creative Commons Attribution 4.0 International license. Further distribution of this work must maintain attribution to the author(s) and the published article's title, journal citation, and DOI. using top quarks in the quark-gluon plasma formed in nucleus-nucleus collisions have also been proposed [4,11]. A good understanding of top quark production in protonnucleus collisions is crucial as a baseline for these studies.

Once produced, the top quark decays promptly without hadronizing (lifetime $c \tau \approx 0.15 \mathrm{fm}$ ) into a $W$ boson plus a bottom quark, and top quark pair events are commonly categorized according to the subsequent decay of the two $W$ bosons. When one $W$ boson decays leptonically ( $\ell \nu$, with $\ell=e, \mu)$ and the other hadronically $\left(q \bar{q}^{\prime}\right)$, the $\ell+$ jets final state presents a typical signature of one isolated charged lepton and momentum imbalance from the unobserved neutrino in one $W$ decay, two light quark jets from the other $W$ decay, and two $b$ jets from the two original top quark decays. Such a final state features a large branching fraction $(\approx 30 \%$ for the $e+$ jets and $\mu+$ jets channels combined, and $\approx 34 \%$ adding also events from the $t \rightarrow$ $W \rightarrow \tau \rightarrow e, \mu$ decay chain) and moderate background contamination, and thereby provides favorable conditions for the detection of $t \bar{t}$ production in proton-nucleus collisions.

This Letter describes the first observation of top quark production in nuclear collisions. The analysis is carried out with $p \mathrm{~Pb}$ collisions collected by the CMS experiment at the LHC at $\sqrt{s_{N N}}=8.16 \mathrm{TeV}$, using $t \bar{t}$ candidates with the event topology described above. The $t \bar{t}$ cross section is extracted from a combined maximum-likelihood fit of the invariant mass of the two light-quark jets from the $W$-boson decay, in different categories of events with zero, one, or at least two $b$-tagged jets.

The central feature of the CMS apparatus is a superconducting solenoid of $6 \mathrm{~m}$ internal diameter, providing a magnetic field of $3.8 \mathrm{~T}$. Within the solenoid volume are a silicon pixel and strip tracker, a lead tungstate crystal electromagnetic calorimeter (ECAL), and a brass and scintillator hadron calorimeter, each composed of a barrel and two end cap sections. Forward calorimeters extend the pseudorapidity coverage provided by the barrel and end cap detectors. Muons are detected in gas-ionization chambers 
embedded in the steel flux-return yoke outside the solenoid. A more detailed description of the CMS detector, together with a definition of the coordinate system used and the relevant kinematic variables, can be found in Ref. [12]. The event sample of $p \mathrm{~Pb}$ collisions collected by the CMS detector in 2016 corresponds to an integrated luminosity of $174 \pm 9 \mathrm{nb}^{-1}$. The lead nuclei and protons had beam energies of 2.56 and $6.5 \mathrm{TeV}$ per nucleon, respectively, corresponding to a nucleon-nucleon center-of-mass energy of $\sqrt{s_{N N}}=8.16 \mathrm{TeV}$. The direction of the proton beam was initially clockwise and was then reversed, producing two data samples of similar size. The pseudorapidity $\eta$ is defined such as to have a positive value in the direction of motion of the proton in both data samples. The number of collisions per bunch crossing is on average 0.5 in the combined data set.

The $p N \rightarrow t \bar{t}+X$ process $(N=p, n)$ is simulated using the PYTHIA 6 Monte Carlo (MC) generator [13] (v.6.424, tune $\mathrm{Z} 2 *[14,15])$ with a mixture of $p p$ and $p n$ interactions corresponding to their ratio in $p \mathrm{~Pb}$ collisions. The number of MC signal events is normalized to the perturbative QCD prediction for the $t \bar{t}$ production cross section at nextto-next-to-leading order (NNLO) with soft gluon resummation at next-to-next-to-leading logarithmic (NNLL) accuracy [2,3], and scaled by the $\mathrm{Pb}$ mass number $A=208$. The value of $m_{t}$ used in all simulated samples is $172.5 \mathrm{GeV}$. Simulated samples of $W+$ jets and Drell-Yan production of charged-lepton pairs with invariant mass larger than $30 \mathrm{GeV}$ are generated using PYTHIA 6. The MC program is used solely for the purpose of efficiency measurements and validation of the functional forms used for the background distributions, as the latter are determined in situ from the data. All PYTHIA signal and background samples are embedded into $p \mathrm{~Pb}$ events generated with EPOS-LHC [16], tuned to reproduce the global $p \mathrm{~Pb}$ event properties experimentally measured, and reconstructed with the same analysis code as used for the data. Because of the different energies of the proton and lead beam, the pseudorapidity for massless particles in the laboratory frame is shifted by 0.465 units in the direction of the proton beam with respect to the $N N$ center-of-mass frame. The kinematics of all MC-generated events are boosted to account for this effect. Simulated samples include an emulation of the full detector response, based on GEANT4 [17], with simulated alignment and calibration conditions tuned on data, and a realistic description of the beam spot, i.e., of the luminous region produced by the collisions.

A two-tier trigger system selects events of interest for off-line analysis [18]. This analysis is restricted to events that fired trigger paths requiring the presence of at least one muon (electron) candidate with transverse momentum (energy) $p_{T}>12 \mathrm{GeV}\left(E_{T}>20 \mathrm{GeV}\right)$. Looser online identification criteria are applied as compared to the offline selection, and no requirement on additional analysis objects is imposed at this level.
Particle candidates are reconstructed off-line with the CMS particle-flow (PF) algorithm [19], which identifies and provides a list of particles using an optimized combination of information from the various elements of the CMS detector. Events are required to contain exactly one muon [20] or electron [21] candidate, with $p_{T}>30 \mathrm{GeV}$ and $|\eta|<2.1$, excluding in the electron case the transition region $1.444<|\eta|<1.566$ between the ECAL barrel and end cap, where the reconstruction of electron objects is less efficient. The muon and electron candidates are required to be isolated from nearby hadronic activity within a cone of $\Delta R=0.3$ around the direction of the track at the primary event vertex. The cone is defined as $\Delta R=\sqrt{(\Delta \eta)^{2}+(\Delta \phi)^{2}}$, and $\Delta \eta$ and $\Delta \phi$ are the separations in pseudorapidity and azimuthal angle. The scalar $p_{T}$ sum of all PF candidates consistent with arising from the primary event vertex and contained within the cone of radius $\Delta R$, excluding the contribution from the lepton candidate, is used to define a relative isolation variable, $I_{\text {rel }}$, through the ratio of this sum to the $p_{T}$ of the lepton candidate. A charged lepton is selected if its relative isolation discriminant value satisfies $I_{\text {rel }}<0.15$ (muon), 0.07 (electron in the barrel), or 0.08 (electron in one of the end caps). These thresholds have been optimized to reduce the contamination from nonprompt leptons. To remove the Drell-Yan background, events are rejected from the analysis if they contain extra electrons (muons) that are reconstructed using a looser set of identification criteria and have $p_{T}>20(15) \mathrm{GeV}$ within $|\eta|<2.5(2.4)$. The efficiency of the lepton selection is measured using a "tag-and-probe" method [22] in events enriched with $Z$-boson candidates and selected by the same trigger requirements as the signal candidate events. The combined reconstruction, lepton identification, and trigger efficiency is determined as a function of lepton $p_{T}$ and $\eta$.

Events are required to have at least four reconstructed jets with $p_{T}>25 \mathrm{GeV}$ and $|\eta|<2.5$, that are separated by at least $\Delta R=0.3$ from the selected muon or electron. Jets are reconstructed from the PF candidates using the anti- $k_{T}$ clustering algorithm [23] with a distance parameter of 0.4 . Jet energy corrections extracted from the full detector simulation are applied as functions of jet $p_{T}$ and $\eta[24,25]$ to both data and simulated samples. A residual correction to the data is applied to account for a small data-MC discrepancy in the jet energy response. Jets from $b$ quarks are tagged based on the presence of a secondary vertex from $B$-hadron decays, identified using a multivariate algorithm combining tracking information [26]. The distinct $t \bar{t}$ signature of two $b$ jets in the event, which rarely occurs in background processes such as $W+$ jets and QCD multijet (collectively labeled as "nontop" background), is used to extract the signal. The number of jets passing a threshold on the $b$-jet identification discriminant, corresponding to a $b$-tagging efficiency of approximately $70 \%$ with a misidentification rate of less than $0.1 \%$ for lightflavor jets, as estimated in simulated $p \mathrm{~Pb}$ events, is used to 
classify the selected events into no $(0 b)$, exactly one $(1 b)$, or at least two $(2 b)$ tagged-jet categories. All three event categories are exploited in a maximum-likelihood fit in order to extract the signal cross section, and simultaneously constrain the background contamination and determine the efficiency of the $b$-jet identification.

In the $\ell+$ jets final state, two light-flavor jets $\left(j j^{\prime}\right)$ are produced in the decay of one of the $W$ bosons, and the resonant nature of their invariant mass provides a distinctive feature of the $t \bar{t}$ signal with respect to the main backgrounds. Given that these light-flavor jets are correlated at production, they are also closer in phase space relative to other dijet combinations in the event. In cases where more than two non- $b$-tagged jets are found, the $j j^{\prime}$ pair with smallest separation in the $\eta-\phi$ plane is used to form a $W$-boson candidate. The invariant mass of those two jets, $m_{j j^{\prime}}$, is used as input for the maximum-likelihood fit.

The parametrization of the signal in the fit model is derived from the MC simulation, while that of the backgrounds is obtained from control regions in the data. In the MC simulation, pairs of jets that are geometrically matched at the parton level with the light quarks coming from $W \rightarrow q \bar{q}^{\prime}$ are marked as "correctly assigned" pairs. The $t \bar{t}$ signal includes correct and wrong assignments. For all mass variables and $b$-jet multiplicity categories, the $m_{j j^{\prime}}$ spectrum is modeled for correct and wrong assignments, respectively, by a Crystal Ball function [27] summed with a gamma function, and by a bifurcated Gaussian (i.e., a Gaussian with different widths to the left and right of its mean) summed with a Landau function [28].

The background contribution from $W+$ jets is assumed to be described by a Landau function, as supported by the agreement observed between the MC simulation and a Landau parametrization in events with no $b$-tagged jets. The background from QCD multijet events due to misidentified or nonprompt leptons satisfying the selection criteria is modeled with the help of dedicated background control regions. In the muon channel, the background region is selected by an inverted isolation requirement, $I_{\text {rel }}>0.2$, while all other selection criteria remain unchanged. In the electron channel, the background shape is modeled with electrons that fail a looser identification requirement. In both cases, the shape of the $m_{j j^{\prime}}$ distribution for this background is estimated with a nonparametric kernel approach [29]. This approach is validated using events with no $b$-tagged jets and with missing transverse momentum (defined as the negative of the vectorial $p_{T}$ sum of all identified particles) smaller than $20 \mathrm{GeV}$ in magnitude. The initial normalization of the QCD multijet backgrounds in the other $b$-jet multiplicity categories is also determined from events with missing transverse momentum smaller than $20 \mathrm{GeV}$.

The number of events in each $b$-jet category is obtained by fitting the sum of the contributions for signal and backgrounds. The free parameters of the fit are the normalization of the signal, QCD multijet, and $W+$ jets yields (as well as the parameters of their functional forms described above), the $b$-finding efficiency, i.e., the probability that a jet originating from the $b$ quark from a top quark decay passes both the kinematic and the $b$-tagging selections, and an overall jet energy scale factor. Figure 1 shows the $m_{j j^{\prime}}$ distribution for events with zero, one, or at least two $b$-tagged jets, compared with the fit results.

To further examine the hypothesis that the selected data are consistent with the production of top quarks, we define a proxy of the top quark mass, $m_{\text {top }}$, as the invariant mass of a $t \rightarrow j j^{\prime} b$ candidate formed by pairing the $W$ candidate with a $b$-tagged jet. This pairing is chosen to minimize the absolute difference between the invariant masses of the $t \rightarrow j j^{\prime} b$ and the $t \rightarrow \ell \nu b$ candidates. In the $0 b$ and $1 b$ categories, the jet(s) with the highest value(s) of the $b$-quark identification discriminator are considered for this purpose. Figure 2 shows the distribution of $m_{\text {top }}$ reconstructed for events in the 0,1 , and $2 b$-tagged jet categories, with all signal and background parameters kept fixed to those from the outcome of the $m_{j j^{\prime}}$ fit.

The total number of $t \bar{t}$ signal events obtained through the fit of the $\mu+$ jets and $e+$ jets channels combined is 710 . Sources of experimental uncertainty in the measurement include the uncertainty in the $b$-tagging efficiency, which is
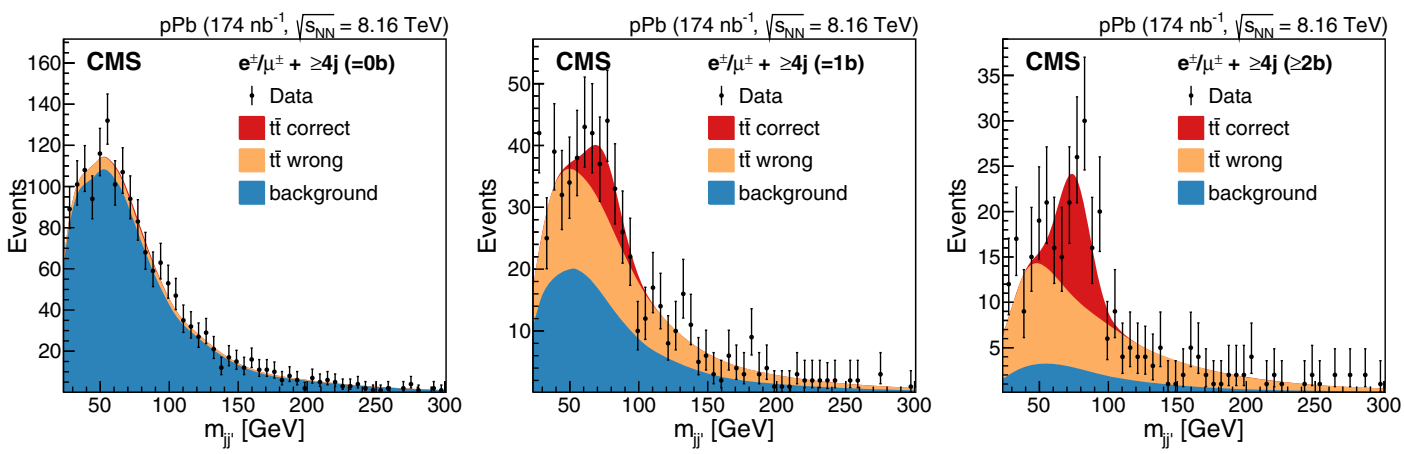

FIG. 1. Invariant mass distributions of the $W$ candidate, $m_{j j^{\prime}}$, in the 0 (left), 1 (center), and 2 (right) $b$-tagged jet categories after all selections. The red and orange areas correspond to the signal simulation (correct and wrong assignments, respectively), while the blue one corresponds to the estimated nontop background contributions. The error bars indicate the statistical uncertainties. 

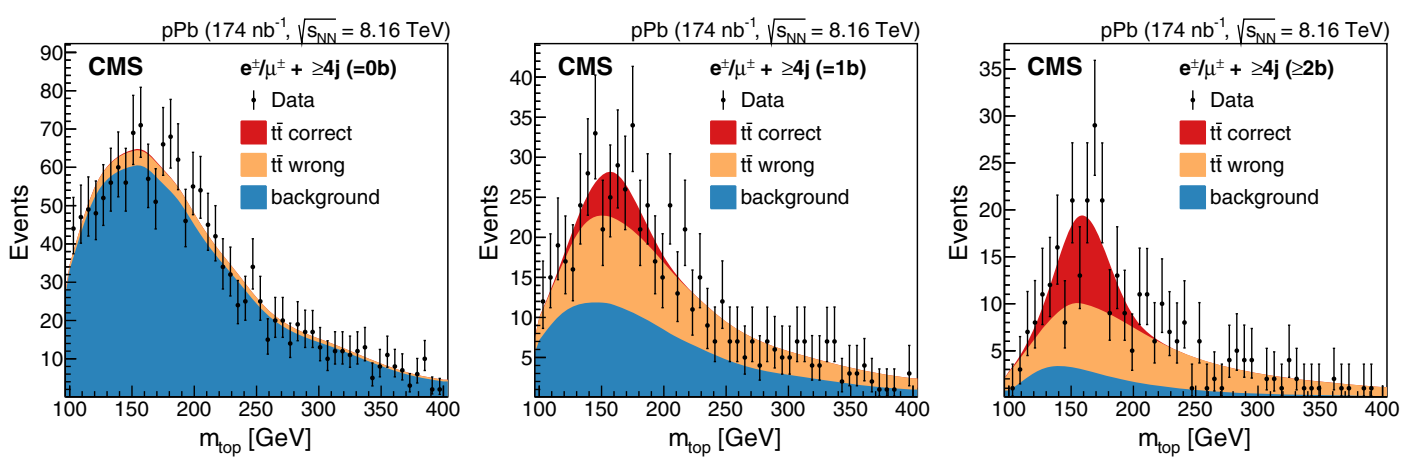

FIG. 2. Invariant mass distributions of the $t \rightarrow j j^{\prime} b$ candidates, $m_{\text {top }}$, in the 0 (left), 1 (center), and 2 (right) $b$-tagged jet categories after all selections. All signal and background parameters are kept fixed to the outcome of the $m_{j j^{\prime}}$ fit. Symbols and patterns are the same as in Fig. 1.

measured in situ and bears the largest effect of $\pm 13 \%$ on the $t \bar{t}$ cross section; and the jet energy scale [24], which takes into account a 3\%-level difference between the reconstructed and generated jet energy in MC events and a 3\% residual calibration uncertainty from data, that together propagate as an additional $\pm 4 \%$ uncertainty in the final cross section. Background shape and normalization uncertainties are also determined in the fit procedure and have a $\pm 7 \%$ effect on the extracted cross section. Uncertainties in the lepton trigger and reconstruction efficiencies, estimated with the tag-and-probe method, result in a $\pm 4 \%$ effect on the measured cross section. The integrated luminosity calibration for $p \mathrm{~Pb}$ data taking conditions results in a $\pm 5 \%$ uncertainty. The jet energy resolution [24], as estimated in proton-proton collision data, and the $0.1 \%$ uncertainty of the LHC beam energy [30], have a numerically insignificant effect on this measurement.

The compatibility of the data with a background-only hypothesis has been evaluated using a profile-likelihood ratio as a test statistic [31], including all systematic uncertainties as nuisance parameters with Gaussian priors. Several tests have been performed, varying the estimation method and the background modeling assumptions. Even with the most conservative assumptions, the backgroundonly hypothesis is excluded with a significance above 5 standard deviations. The $t \bar{t}$ production cross section is then obtained via

$$
\sigma_{\bar{t} \bar{t}}=\frac{S}{\mathcal{A} \mathcal{E} \mathcal{L}}
$$

where $S$ is the number of fitted signal events; $\mathcal{A}=0.060 \pm$ 0.002 and $0.056 \pm 0.002$ are the total acceptances in the $\mu+$ jets and $e+$ jets channels relative to all generated $t \bar{t}$ events, including the branching fraction to leptons, as determined from simulation; $\varepsilon=0.91 \pm 0.04$ and $0.63 \pm$ 0.03 are the $\mu+$ jets and $e+$ jets event selection efficiencies as estimated from data; and $\mathcal{L}$ is the total integrated luminosity. The $4 \%$ uncertainty in the acceptance correction $\mathcal{A}$, including its dependence on the proton and $\mathrm{Pb} \mathrm{PDF}$, and on the values of theoretical scales and the QCD coupling $\left(\alpha_{s}=0.118 \pm 0.001\right.$ at the $Z$-boson pole mass), has been determined from a NLO $p \mathrm{~Pb} \rightarrow$ $t \bar{t}+X$ sample generated with POWHEG (v.2) [32-34]. The total uncertainty on $S$ is obtained from the covariance matrix of the fit. It is further split into a statistical part, by leaving $\sigma_{t \bar{t}}$ to float in the fit and fixing all other parameters to their post-fit values, and a systematic part, by subtracting the square of the statistical uncertainty from the square of the total uncertainty. From Eq. (1), we measure

$$
\begin{aligned}
& \sigma_{t \bar{t}}^{\mu+\text { jets }}=44 \pm 3(\text { stat }) \pm 8(\text { syst }) \mathrm{nb}, \\
& \sigma_{t \bar{t}}^{e+\mathrm{jets}}=56 \pm 4(\text { stat }) \pm 13 \text { (syst) nb, }
\end{aligned}
$$

in the individual $\mu+$ jets $(S=420)$ and $e+$ jets $(S=348)$ channels, with relative total uncertainties of $18 \%$ and $23 \%$, respectively. The combined fit to both channels yields

$$
\sigma_{\bar{t} \bar{t}}=45 \pm 8(\text { total }) \mathrm{nb} .
$$

The measured cross section is found to be consistent with the theoretical prediction [5] $\sigma(p \mathrm{~Pb} \rightarrow t \bar{t}+X)=59.0 \pm$ $5.3(\mathrm{PDF})_{-2.1}^{+1.6}$ (scale) nb, computed with MCFM (v.8) [35] using the CT14 proton PDF [36] and the EPPS16 nPDF for the lead ions [8], scaled to NNLO + NNLL accuracy with a $K$ factor computed with TOP++ (v.2.0) [2], and multiplied by $A=208$. The PDF uncertainties are obtained from the corresponding $56+40$ eigenvalues of the CT14 + EPPS16 sets (corresponding to a $90 \%$ confidence level) added in quadrature, while the theoretical scale uncertainty is estimated by modifying the factorization and renormalization scales within a factor of 2 with respect to their default value set at $\mu_{F}=\mu_{R}=m_{t}$. The same calculation with the CT10 proton PDF [37] and EPS09 [7] nPDF yields $\sigma(p \mathrm{~Pb} \rightarrow t \bar{t}+X)=57.5 \pm_{-3.3}^{+4.3}(\mathrm{PDF})_{-2.0}^{+1.5}($ scale $) \mathrm{nb}$. The difference in the theoretical $t \bar{t}$ cross section computed with the PDF for free protons and for bound nucleons is small. A net overall antishadowing effect increases the total topquark pair cross section by only $4 \%$ for both the EPPS 16 and EPS09 sets in $p \mathrm{~Pb}$ relative to $p p$ collisions [5]. Such a 


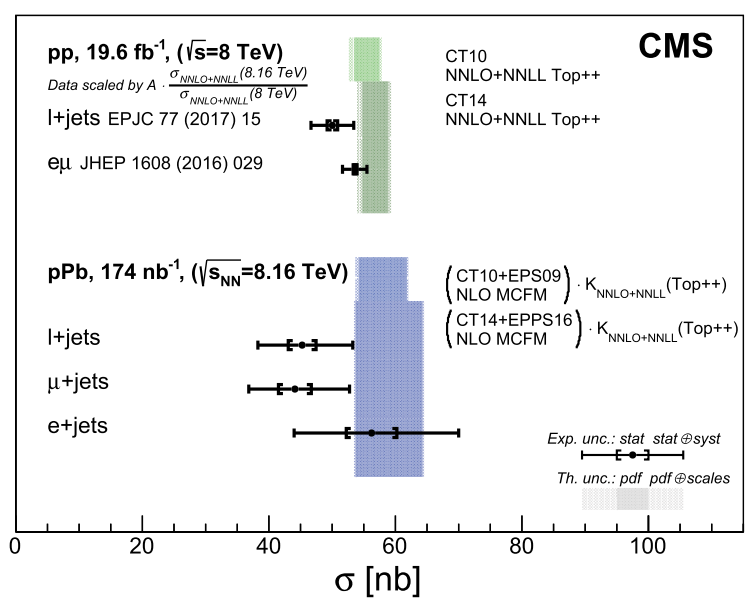

FIG. 3. Total $t \bar{t}$ cross sections measured in the $e+$ jets, $\mu+$ jets, and combined $\ell+$ jets channels in $p \mathrm{~Pb}$ collisions at $\sqrt{s_{N N}}=$ $8.16 \mathrm{TeV}$, compared to theoretical NNLO + NNLL predictions, and to scaled $\sqrt{s}=8 \mathrm{TeV} p p$ results $[38,39]$. The total experimental error bars (theoretical error bands) include statistical and systematic (PDF and scale) uncertainties added in quadrature.

difference is too small to be observed in the data with the current experimental uncertainties. Figure 3 shows the measured and theoretical cross sections for $t \bar{t}$ production in $p \mathrm{~Pb}$ collisions at $\sqrt{s_{N N}}=8.16 \mathrm{TeV}$, compared with the results from $p p$ collisions at $\sqrt{s}=8 \mathrm{TeV}[38,39]$ scaled by $A$ and by the ratio of $8.16 \mathrm{TeV}$ over $8 \mathrm{TeV} \mathrm{NNLO}+$ NNLL cross sections.

In summary, the top pair production cross section has been measured for the first time in proton-nucleus collisions, using $p \mathrm{~Pb}$ data at $\sqrt{s_{N N}}=8.16 \mathrm{TeV}$ with a total integrated luminosity of $174 \mathrm{nb}^{-1}$. The measurement is performed by analyzing events with exactly one isolated electron or muon and at least four jets. The significance of the $t \bar{t}$ signal against the background-only hypothesis is above 5 standard deviations. The measured cross section is $\sigma_{t \bar{t}}=45 \pm 8 \mathrm{nb}$, consistent with the expectations from scaled $p p$ data as well as perturbative quantum chromodynamics calculations. This first measurement paves the way for further detailed investigations of top-quark production in nuclear interactions, providing in particular a new tool for studies of the strongly interacting matter created in nucleus-nucleus collisions.

We congratulate our colleagues in the CERN accelerator departments for the excellent performance of the LHC and thank the technical and administrative staffs at CERN and at other CMS institutes for their contributions to the success of the CMS effort. In addition, we gratefully acknowledge the computing centers and personnel of the Worldwide LHC Computing Grid for delivering so effectively the computing infrastructure essential to our analyses. Finally, we acknowledge the enduring support for the construction and operation of the LHC and the CMS detector provided by the following funding agencies: BMWFW and FWF (Austria); FNRS and FWO (Belgium); CNPq, CAPES, FAPERJ, and FAPESP (Brazil); MES (Bulgaria); CERN; CAS, MoST, and NSFC (China); COLCIENCIAS (Colombia); MSES and CSF (Croatia); RPF (Cyprus); SENESCYT (Ecuador); MoER, ERC IUT, and ERDF (Estonia); Academy of Finland, MEC, and HIP (Finland); CEA and CNRS/IN2P3 (France); BMBF, DFG, and HGF (Germany); GSRT (Greece); OTKA and NIH (Hungary); DAE and DST (India); IPM (Iran); SFI (Ireland); INFN (Italy); MSIP and NRF (Republic of Korea); LAS (Lithuania); MOE and UM (Malaysia); BUAP, CINVESTAV, CONACYT, LNS, SEP, and UASLP-FAI (Mexico); MBIE (New Zealand); PAEC (Pakistan); MSHE and NSC (Poland); FCT (Portugal); JINR (Dubna); MON, RosAtom, RAS, RFBR and RAEP (Russia); MESTD (Serbia); SEIDI, CPAN, PCTI and FEDER (Spain); Swiss Funding Agencies (Switzerland); MST (Taipei); ThEPCenter, IPST, STAR, and NSTDA (Thailand); TUBITAK and TAEK (Turkey); NASU and SFFR (Ukraine); STFC (United Kingdom); DOE and NSF (USA).

State Fund for Fundamental Researches

[1] C. Patrignani et al. (Particle Data Group), Review of particle physics, Chin. Phys. C 40, 100001 (2016).

[2] M. Czakon and A. Mitov, TOP++: a program for the calculation of the top-pair cross-section at hadron colliders, Comput. Phys. Commun. 185, 2930 (2014).

[3] M. Czakon, P. Fiedler, and A. Mitov, Total Top-Quark PairProduction Cross Section at Hadron Colliders Through $\mathrm{O}\left(\alpha_{S}^{4}\right)$, Phys. Rev. Lett. 110, 252004 (2013).

[4] D. d'Enterria, K. Krajczár, and H. Paukkunen, Top-quark production in proton-nucleus and nucleus-nucleus collisions at LHC energies and beyond, Phys. Lett. B 746, 64 (2015).

[5] D. d'Enterria, Top-quark pair production cross sections at $\mathrm{NNLO}+\mathrm{NNLL}$ in $p \mathrm{~Pb}$ collisions at $\sqrt{s_{N N}}=8.16 \mathrm{TeV}$, arXiv:1706.09521 [Nucl. Phys. A (to be published)].

[6] J. J. Aubert et al. (European Muon Collaboration), The ratio of the nucleon structure functions $F_{2}^{N}$ for iron and deuterium, Phys. Lett. B 123, 275 (1983).

[7] K. J. Eskola, H. Paukkunen, and C. A. Salgado, EPS09: A new generation of NLO and LO nuclear parton distribution functions, J. High Energy Phys. 04 (2009) 065.

[8] K. J. Eskola, P. Paakkinen, H. Paukkunen, and C. A. Salgado, EPPS16: Nuclear parton distributions with LHC data, Eur. Phys. J. C 77, 163 (2017).

[9] CMS Collaboration, Study of $W$ boson production in $p \mathrm{~Pb}$ collisions at $\sqrt{s_{N N}}=5.02 \mathrm{TeV}$, Phys. Lett. B 750, 565 (2015).

[10] CMS Collaboration, Study of $Z$ boson production in $p \mathrm{~Pb}$ collisions at $\sqrt{s_{N N}}=5.02 \mathrm{TeV}$, Phys. Lett. B 759, 36 (2016).

[11] A. Dainese et al., Heavy ions at the future circular collider, CERN Yellow Report No. CERN-TH-2016-107, 2017, 
https://e-publishing.cern.ch/index.php/CYRM/article/view/ 515.

[12] CMS Collaboration, The CMS experiment at the CERN LHC, J. Instrum. 3, S08004 (2008).

[13] T. Sjöstrand, S. Mrenna, and P. Skands, PYTHIA 6.4 physics and manual, J. High Energy Phys. 05 (2006) 026.

[14] CMS Collaboration, Study of the underlying event at forward rapidity in $p p$ collisions at $\sqrt{s}=0.9,2.76$, and 7 TeV, J. High Energy Phys. 04 (2013) 072.

[15] CMS Collaboration, Event generator tunes obtained from underlying event and multiparton scattering measurements, Eur. Phys. J. C 76, 155 (2016).

[16] T. Pierog, I. Karpenko, J. M. Katzy, E. Yatsenko, and K. Werner, EPOS-LHC: Test of collective hadronization with data measured at the CERN Large Hadron Collider, Phys. Rev. C 92, 034906 (2015).

[17] S. Agostinelli et al. (GEANT4 Collaboration), GEANT4: A simulation toolkit, Nucl. Instrum. Methods Phys. Res., Sect. A 506, 250 (2003).

[18] CMS Collaboration, The CMS trigger system, J. Instrum. 12, P01020 (2017).

[19] CMS Collaboration, Particle-flow reconstruction and global event description with the CMS detector, J. Instrum. 12, P10003 (2017).

[20] CMS Collaboration, The performance of the CMS muon detector in proton-proton collisions at $\sqrt{s}=7 \mathrm{TeV}$ at the LHC, J. Instrum. 8, P11002 (2013).

[21] CMS Collaboration, Performance of electron reconstruction and selection with the CMS detector in proton-proton collisions at $\sqrt{s}=8 \mathrm{TeV}$, J. Instrum. 10, P06005 (2015).

[22] CMS Collaboration, Measurements of inclusive $W$ and $Z$ cross sections in $p p$ collisions at $\sqrt{s}=7 \mathrm{TeV}$, J. High Energy Phys. 01 (2011) 080.

[23] M. Cacciari, G. P. Salam, and G. Soyez, The anti- $k_{t}$ jet clustering algorithm, J. High Energy Phys. 04 (2008) 063.

[24] CMS Collaboration, Determination of jet energy calibration and transverse momentum resolution in CMS, J. Instrum. 6 , P11002 (2011).

[25] CMS Collaboration, Jet energy scale and resolution in the CMS experiment in $p p$ collisions at $8 \mathrm{TeV}$, J. Instrum. 12, P02014 (2017).

[26] CMS Collaboration, Identification of $b$-quark jets with the CMS experiment, J. Instrum. 8, P04013 (2013).

[27] M. J. Oreglia, Ph.D. thesis, Stanford University, 1980; SLAC Report No. SLAC-R-236, see Appendix D.
[28] W. Verkerke and D.P. Kirkby, The ROOFIT toolkit for data modeling, Proceedings of the 13th International Conference for Computing in High-Energy Physics and Nuclear Physics (CHEP 2003), March 2003, La Jolla, California, USA (2003) [arXiv:physics/0306116].

[29] K. S. Cranmer, Kernel estimation in high-energy physics, Comput. Phys. Commun. 136, 198 (2001).

[30] E. Todesco and J. Wenninger, Large Hadron Collider momentum calibration and accuracy, Phys. Rev. Accel. Beams 20, 081003 (2017).

[31] ATLAS and CMS Collaborations, Procedure for the LHC Higgs boson search combination in summer 2011, ATLAS Note No. ATL-PHYS-PUB-2011-011 and CMS Note No. CMS-NOTE-2011-005, 2011, https://cds.cern.ch/ record/1379837.

[32] S. Frixione, P. Nason, and C. Oleari, Matching NLO QCD computations with parton shower simulations: The POWHEG method, J. High Energy Phys. 11 (2007) 070.

[33] S. Alioli, P. Nason, C. Oleari, and E. Re, A general framework for implementing NLO calculations in shower Monte Carlo programs: The POWHEG BOX, J. High Energy Phys. 06 (2010) 043.

[34] S. Frixione, P. Nason, and G. Ridolfi, A positive-weight next-to-leading-order Monte Carlo for heavy flavour hadroproduction, J. High Energy Phys. 09 (2007) 126.

[35] J. M. Campbell and R. K. Ellis, MCFM for the Tevatron and the LHC, Nucl. Phys. B, Proc. Suppl. 205-206, 10 (2010).

[36] S. Dulat, T.-J. Hou, J. Gao, M. Guzzi, J. Huston, P. Nadolsky, J. Pumplin, C. Schmidt, D. Stump, and C. P. Yuan, New parton distribution functions from a global analysis of quantum chromodynamics, Phys. Rev. D 93, 033006 (2016).

[37] J. Gao, M. Guzzi, J. Huston, H.-L. Lai, Z. Li, P. Nadolsky, J. Pumplin, D. Stump, and C. P. Yuan, CT10 next-to-next-toleading order global analysis of QCD, Phys. Rev. D 89, 033009 (2014).

[38] CMS Collaboration, Measurement of the $t \bar{t}$ production cross section in the $\mathrm{e} \mu$ channel in proton-proton collisions at $\sqrt{s}=7$ and $8 \mathrm{TeV}$, J. High Energy Phys. 08 (2016) 029.

[39] CMS Collaboration, Measurements of the $t \bar{t}$ production cross section in lepton + jets final states in $p p$ collisions at $8 \mathrm{TeV}$ and ratio of 8 to $7 \mathrm{TeV}$ cross sections, Eur. Phys. J. C 77, 15 (2017).

A. M. Sirunyan, ${ }^{1}$ A. Tumasyan, ${ }^{1}$ W. Adam, ${ }^{2}$ F. Ambrogi, ${ }^{2}$ E. Asilar, ${ }^{2}$ T. Bergauer, ${ }^{2}$ J. Brandstetter, ${ }^{2}$ E. Brondolin, ${ }^{2}$ M. Dragicevic, ${ }^{2}$ J. Erö, ${ }^{2}$ A. Escalante Del Valle, ${ }^{2}$ M. Flechl, ${ }^{2}$ M. Friedl, ${ }^{2}$ R. Frühwirth, ${ }^{2, b}$ V. M. Ghete, ${ }^{2}$ J. Grossmann, ${ }^{2}$ J. Hrubec, ${ }^{2}$ M. Jeitler, ${ }^{2, b}$ A. König, ${ }^{2}$ N. Krammer, ${ }^{2}$ I. Krätschmer, ${ }^{2}$ D. Liko, ${ }^{2}$ T. Madlener, ${ }^{2}$ I. Mikulec, ${ }^{2}$ E. Pree, ${ }^{2}$ N. Rad, ${ }^{2}$ H. Rohringer, ${ }^{2}$ J. Schieck, ${ }^{2, b}$ R. Schöfbeck, ${ }^{2}$ M. Spanring, ${ }^{2}$ D. Spitzbart, ${ }^{2}$ W. Waltenberger, ${ }^{2}$ J. Wittmann, ${ }^{2}$ C.-E. Wulz, ${ }^{2, b}$ M. Zarucki, ${ }^{2}$ V. Chekhovsky, ${ }^{3}$ V. Mossolov, ${ }^{3}$ J. Suarez Gonzalez, ${ }^{3}$ E. A. De Wolf, ${ }^{4}$ D. Di Croce, ${ }^{4}$ X. Janssen, ${ }^{4}$ J. Lauwers, ${ }^{4}$ M. Van De Klundert, ${ }^{4}$ H. Van Haevermaet, ${ }^{4}$ P. Van Mechelen, ${ }^{4}$ N. Van Remortel, ${ }^{4}$ S. Abu Zeid, ${ }^{5}$ F. Blekman, ${ }^{5}$ J. D'Hondt, ${ }^{5}$ I. De Bruyn, ${ }^{5}$ J. De Clercq, ${ }^{5}$ K. Deroover, ${ }^{5}$ G. Flouris, ${ }^{5}$ D. Lontkovskyi,${ }^{5}$ S. Lowette, ${ }^{5}$ I. Marchesini, ${ }^{5}$ S. Moortgat, ${ }^{5}$ L. Moreels, ${ }^{5}$ Q. Python, ${ }^{5}$ K. Skovpen, ${ }^{5}$ S. Tavernier, ${ }^{5}$ W. Van Doninck, ${ }^{5}$ P. Van Mulders, ${ }^{5}$ I. Van Parijs, ${ }^{5}$ D. Beghin, ${ }^{6}$ B. Bilin, ${ }^{6}$ H. Brun, ${ }^{6}$ B. Clerbaux, ${ }^{6}$ G. De Lentdecker, ${ }^{6}$ H. Delannoy, ${ }^{6}$ B. Dorney, ${ }^{6}$ G. Fasanella, ${ }^{6}$ L. Favart, ${ }^{6}$ R. Goldouzian, ${ }^{6}$ 
A. Grebenyuk, ${ }^{6}$ A. K. Kalsi, ${ }^{6}$ T. Lenzi, ${ }^{6}$ J. Luetic, ${ }^{6}$ T. Maerschalk, ${ }^{6}$ A. Marinov, ${ }^{6}$ T. Seva, ${ }^{6}$ E. Starling, ${ }^{6}$ C. Vander Velde, ${ }^{6}$ P. Vanlaer, ${ }^{6}$ D. Vannerom, ${ }^{6}$ R. Yonamine, ${ }^{6}$ F. Zenoni, ${ }^{6}$ T. Cornelis, ${ }^{7}$ D. Dobur, ${ }^{7}$ A. Fagot, ${ }^{7}$ M. Gul, ${ }^{7}$ I. Khvastunov, ${ }^{7, c}$ D. Poyraz, ${ }^{7}$ C. Roskas, ${ }^{7}$ S. Salva, ${ }^{7}$ M. Tytgat,${ }^{7}$ W. Verbeke, ${ }^{7}$ N. Zaganidis, ${ }^{7}$ H. Bakhshiansohi, ${ }^{8}$ O. Bondu, ${ }^{8}$ S. Brochet, ${ }^{8}$ G. Bruno, ${ }^{8}$ C. Caputo, ${ }^{8}$ A. Caudron,${ }^{8}$ P. David,${ }^{8}$ S. De Visscher, ${ }^{8}$ C. Delaere, ${ }^{8}$ M. Delcourt, ${ }^{8}$ B. Francois,${ }^{8}$ A. Giammanco, ${ }^{8}$ M. Komm, ${ }^{8}$ G. Krintiras, ${ }^{8}$ V. Lemaitre, ${ }^{8}$ A. Magitteri, ${ }^{8}$ A. Mertens, ${ }^{8}$ M. Musich, ${ }^{8}$ K. Piotrzkowski, ${ }^{8}$ L. Quertenmont, ${ }^{8}$ A. Saggio, ${ }^{8}$ M. Vidal Marono, ${ }^{8}$ S. Wertz, ${ }^{8}$ J. Zobec, ${ }^{8}$ W. L. Aldá Júnior, ${ }^{9}$ F. L. Alves, ${ }^{9}$ G. A. Alves, ${ }^{9}$ L. Brito, ${ }^{9}$ M. Correa Martins Junior, ${ }^{9}$ C. Hensel, ${ }^{9}$ A. Moraes, ${ }^{9}$ M. E. Pol,${ }^{9}$ P. Rebello Teles, ${ }^{9}$ E. Belchior Batista Das Chagas, ${ }^{10}$ W. Carvalho, ${ }^{10}$ J. Chinellato, ${ }^{10, \mathrm{~d}}$ E. Coelho, ${ }^{10}$ E. M. Da Costa, ${ }^{10}$ G. G. Da Silveira, ${ }^{10, \mathrm{e}}$ D. De Jesus Damiao, ${ }^{10}$ S. Fonseca De Souza, ${ }^{10}$ L. M. Huertas Guativa, ${ }^{10}$ H. Malbouisson, ${ }^{10}$ M. Melo De Almeida, ${ }^{10}$ C. Mora Herrera, ${ }^{10}$ L. Mundim, ${ }^{10}$ H. Nogima, ${ }^{10}$ L. J. Sanchez Rosas, ${ }^{10}$ A. Santoro, ${ }^{10}$ A. Sznajder, ${ }^{10}$ M. Thiel, ${ }^{10}$ E. J. Tonelli Manganote, ${ }^{10, d}$ F. Torres Da Silva De Araujo, ${ }^{10}$ A. Vilela Pereira, ${ }^{10}$ S. Ahuja, ${ }^{11 \mathrm{a}}$ C. A. Bernardes, ${ }^{11 \mathrm{a}}$ T. R. Fernandez Perez Tomei, ${ }^{11 \mathrm{a}}$ E. M. Gregores ${ }^{11 \mathrm{~b}}$ P. G. Mercadante, ${ }^{11 \mathrm{~b}}$ S. F. Novaes, ${ }^{11 \mathrm{a}}$ Sandra S. Padula, ${ }^{11 \mathrm{a}}$ D. Romero Abad, ${ }^{11 \mathrm{~b}}$ J. C. Ruiz Vargas, ${ }^{1 \mathrm{a}}$ A. Aleksandrov, ${ }^{12}$ R. Hadjiiska, ${ }^{12}$ P. Iaydjiev, ${ }^{12}$ M. Misheva, ${ }^{12}$ M. Rodozov,${ }^{12}$ M. Shopova, ${ }^{12}$ G. Sultanov, ${ }^{12}$ A. Dimitrov, ${ }^{13}$ L. Litov, ${ }^{13}$ B. Pavlov, ${ }^{13}$ P. Petkov, ${ }^{13}$ W. Fang, ${ }^{14, f}$ X. Gao, ${ }^{14, f}$ L. Yuan, ${ }^{14}$ M. Ahmad, ${ }^{15}$ J. G. Bian, ${ }^{15}$ G. M. Chen, ${ }^{15}$ H. S. Chen, ${ }^{15}$ M. Chen, ${ }^{15}$ Y. Chen, ${ }^{15}$ C. H. Jiang, ${ }^{15}$ D. Leggat, ${ }^{15}$ H. Liao, ${ }^{15}$ Z. Liu, ${ }^{15}$ F. Romeo, ${ }^{15}$ S. M. Shaheen, ${ }^{15}$ A. Spiezia, ${ }^{15}$ J. Tao, ${ }^{15}$ C. Wang, ${ }^{15}$ Z. Wang, ${ }^{15}$ E. Yazgan, ${ }^{15}$ H. Zhang, ${ }^{15}$ S. Zhang, ${ }^{15}$ J. Zhao, ${ }^{15}$ Y. Ban, ${ }^{16}$ G. Chen, ${ }^{16}$ J. Li, ${ }^{16}$ Q. Li, ${ }^{16}$ S. Liu, ${ }^{16}$ Y. Mao, ${ }^{16}$ S. J. Qian, ${ }^{16}$ D. Wang,${ }^{16}$ Z. Xu, ${ }^{16}$ F. Zhang, ${ }^{16, f}$ Y. Wang, ${ }^{17}$ C. Avila, ${ }^{18}$ A. Cabrera, ${ }^{18}$ L. F. Chaparro Sierra, ${ }^{18}$ C. Florez ${ }^{18}$ C. F. González Hernández, ${ }^{18}$ J. D. Ruiz Alvarez, ${ }^{18}$ M. A. Segura Delgado, ${ }^{18}$ B. Courbon, ${ }^{19}$ N. Godinovic, ${ }^{19}$ D. Lelas, ${ }^{19}$ I. Puljak, ${ }^{19}$ P. M. Ribeiro Cipriano, ${ }^{19}$ T. Sculac, ${ }^{19}$ Z. Antunovic,${ }^{20}$ M. Kovac, ${ }^{20}$ V. Brigljevic, ${ }^{21}$ D. Ferencek, ${ }^{21}$ K. Kadija, ${ }^{21}$ B. Mesic, ${ }^{21}$ A. Starodumov, ${ }^{21, g}$ T. Susa, ${ }^{21}$ M. W. Ather, ${ }^{22}$ A. Attikis, ${ }^{22}$ G. Mavromanolakis, ${ }^{22}$ J. Mousa, ${ }^{22}$ C. Nicolaou, ${ }^{22}$ F. Ptochos, ${ }^{22}$ P. A. Razis, ${ }^{22}$ H. Rykaczewski, ${ }^{22}$ M. Finger, ${ }^{23, h}$ M. Finger Jr., ${ }^{23, h}$ E. Carrera Jarrin, ${ }^{24}$ Y. Assran, ${ }^{25, i, j}$ S. Elgammal, ${ }^{25, j}$ A. Mahrous, ${ }^{25, k}$ R. K. Dewanjee, ${ }^{26}$ M. Kadastik, ${ }^{26}$ L. Perrini ${ }^{26}$ M. Raidal, ${ }^{26}$ A. Tiko, ${ }^{26}$ C. Veelken, ${ }^{26}$ P. Eerola,${ }^{27}$ H. Kirschenmann, ${ }^{27}$ J. Pekkanen, ${ }^{27}$ M. Voutilainen, ${ }^{27}$ J. Havukainen, ${ }^{28}$ J. K. Heikkilä, ${ }^{28}$ T. Järvinen, ${ }^{28}$ V. Karimäki, ${ }^{28}$ R. Kinnunen, ${ }^{28}$ T. Lampén, ${ }^{28}$ K. Lassila-Perini, ${ }^{28}$ S. Laurila, ${ }^{28}$ S. Lehti, ${ }^{28}$ T. Lindén, ${ }^{28}$ P. Luukka, ${ }^{28}$ H. Siikonen, ${ }^{28}$ E. Tuominen, ${ }^{28}$ J. Tuominiemi, ${ }^{28}$ T. Tuuva, ${ }^{29}$ M. Besancon, ${ }^{30}$ F. Couderc, ${ }^{30}$ M. Dejardin, ${ }^{30}$ D. Denegri, ${ }^{30}$ J. L. Faure, ${ }^{30}$ F. Ferri, ${ }^{30}$ S. Ganjour, ${ }^{30}$ S. Ghosh,${ }^{30}$ P. Gras,${ }^{30}$ G. Hamel de Monchenault,${ }^{30}$ P. Jarry,${ }^{30}$ I. Kucher, ${ }^{30}$ C. Leloup, ${ }^{30}$ E. Locci, ${ }^{30}$ M. Machet,${ }^{30}$ J. Malcles, ${ }^{30}$ G. Negro, ${ }^{30}$ J. Rander, ${ }^{30}$ A. Rosowsky, ${ }^{30}$ M. Ö. Sahin, ${ }^{30}$ M. Titov, ${ }^{30}$ A. Abdulsalam, ${ }^{31,1}$ C. Amendola, ${ }^{31}$ I. Antropov, ${ }^{31}$ S. Baffioni, ${ }^{31}$ F. Beaudette, ${ }^{31}$ P. Busson, ${ }^{31}$ L. Cadamuro, ${ }^{31}$ C. Charlot, ${ }^{31}$ R. Granier de Cassagnac, ${ }^{31}$ M. Jo, ${ }^{31}$ S. Lisniak,${ }^{31}$ A. Lobanov,${ }^{31}$ J. Martin Blanco, ${ }^{31}$ M. Nguyen, ${ }^{31}$ C. Ochando, ${ }^{31}$ G. Ortona, ${ }^{31}$ P. Paganini, ${ }^{31}$ P. Pigard, ${ }^{31}$ R. Salerno, ${ }^{31}$ J. B. Sauvan, ${ }^{31}$ Y. Sirois, ${ }^{31}$ A. G. Stahl Leiton, ${ }^{31}$ T. Strebler, ${ }^{31}$ Y. Yilmaz, ${ }^{31}$ A. Zabi, ${ }^{31}$ A. Zghiche, ${ }^{31}$ J.-L. Agram, ${ }^{32, m}$ J. Andrea, ${ }^{32}$ D. Bloch, ${ }^{32}$ J.-M. Brom, ${ }^{32}$ M. Buttignol, ${ }^{32}$ E. C. Chabert, ${ }^{32}$ N. Chanon, ${ }^{32}$ C. Collard, ${ }^{32}$ E. Conte, ${ }^{32, m}$ X. Coubez, ${ }^{32}$ J.-C. Fontaine, ${ }^{32, \mathrm{~m}}$ D. Gelé, ${ }^{32}$ U. Goerlach, ${ }^{32}$ M. Jansová, ${ }^{32}$ A.-C. Le Bihan, ${ }^{32}$ N. Tonon, ${ }^{32}$ P. Van Hove, ${ }^{32}$ S. Gadrat ${ }^{33}$ S. Beauceron, ${ }^{34}$ C. Bernet ${ }^{34}$ G. Boudoul,${ }^{34}$ R. Chierici, ${ }^{34}$ D. Contardo,${ }^{34}$ P. Depasse ${ }^{34}$ H. El Mamouni, ${ }^{34}$ J. Fay,${ }^{34}$ L. Finco, ${ }^{34}$ S. Gascon, ${ }^{34}$ M. Gouzevitch, ${ }^{34}$ G. Grenier, ${ }^{34}$ B. Ille, ${ }^{34}$ F. Lagarde,${ }^{34}$ I. B. Laktineh,${ }^{34}$ M. Lethuillier, ${ }^{34}$ L. Mirabito, ${ }^{34}$ A. L. Pequegnot, ${ }^{34}$ S. Perries, ${ }^{34}$ A. Popov, ${ }^{34, n}$ V. Sordini, ${ }^{34}$ M. Vander Donckt, ${ }^{34}$ S. Viret,,${ }^{34}$ T. Toriashvili, ${ }^{35,0}$ I. Bagaturia, ${ }^{36, p}$ C. Autermann, ${ }^{37}$ L. Feld,${ }^{37}$ M. K. Kiesel,${ }^{37}$ K. Klein, ${ }^{37}$ M. Lipinski, ${ }^{37}$ M. Preuten, ${ }^{37}$ C. Schomakers,${ }^{37}$ J. Schulz,${ }^{37}$ M. Teroerde, ${ }^{37}$ V. Zhukov, ${ }^{37, n}$ A. Albert, ${ }^{38}$ E. Dietz-Laursonn, ${ }^{38}$ D. Duchardt, ${ }^{38}$ M. Endres, ${ }^{38}$ M. Erdmann, ${ }^{38}$ S. Erdweg, ${ }^{38}$ T. Esch,${ }^{38}$ R. Fischer, ${ }^{38}$ A. Güth, ${ }^{38}$ M. Hamer, ${ }^{38}$ T. Hebbeker,${ }^{38}$ C. Heidemann, ${ }^{38}$ K. Hoepfner, ${ }^{38}$ S. Knutzen, ${ }^{38}$ M. Merschmeyer, ${ }^{38}$ A. Meyer, ${ }^{38}$ P. Millet, ${ }^{38}$ S. Mukherjee, ${ }^{38}$ T. Pook, ${ }^{38}$ M. Radziej, ${ }^{38}$ H. Reithler, ${ }^{38}$ M. Rieger, ${ }^{38}$ F. Scheuch,$^{38}$ D. Teyssier, ${ }^{38}$ S. Thüer,${ }^{38}$ G. Flügge, ${ }^{39}$ B. Kargoll, ${ }^{39}$ T. Kress,${ }^{39}$ A. Künsken, ${ }^{39}$ T. Müller, ${ }^{39}$ A. Nehrkorn, ${ }^{39}$ A. Nowack, ${ }^{39}$ C. Pistone,${ }^{39}$ O. Pooth ${ }^{39}$ A. Stahl,${ }^{39, q}$ M. Aldaya Martin, ${ }^{40}$ T. Arndt, ${ }^{40}$ C. Asawatangtrakuldee, ${ }^{40}$ K. Beernaert, ${ }^{40}$ O. Behnke, ${ }^{40}$ U. Behrens, ${ }^{40}$ A. Bermúdez Martínez,${ }^{40}$ A. A. Bin Anuar, ${ }^{40}$ K. Borras,${ }^{40, r}$ V. Botta, ${ }^{40}$ A. Campbell, ${ }^{40}$ P. Connor, ${ }^{40}$ C. Contreras-Campana,${ }^{40}$ F. Costanza, ${ }^{40}$ C. Diez Pardos,${ }^{40}$ G. Eckerlin, ${ }^{40}$ D. Eckstein, ${ }^{40}$ T. Eichhorn ${ }^{40}$ E. Eren,${ }^{40}$ E. Gallo, ${ }^{40, s}$ J. Garay Garcia, ${ }^{40}$ A. Geiser, ${ }^{40}$ J. M. Grados Luyando, ${ }^{40}$ A. Grohsjean, ${ }^{40}$ P. Gunnellini, ${ }^{40}$ M. Guthoff, ${ }^{40}$ A. Harb, ${ }^{40}$ J. Hauk,${ }^{40}$ M. Hempel, ${ }^{40, t}$ H. Jung, ${ }^{40}$ M. Kasemann, ${ }^{40}$ J. Keaveney, ${ }^{40}$ C. Kleinwort, ${ }^{40}$ I. Korol, ${ }^{40}$ D. Krücker, ${ }^{40}$ W. Lange, ${ }^{40}$ A. Lelek, ${ }^{40}$ T. Lenz,${ }^{40}$ J. Leonard, ${ }^{40}$ K. Lipka, ${ }^{40}$ W. Lohmann, ${ }^{40, t}$ R. Mankel, ${ }^{40}$ I.-A. Melzer-Pellmann, ${ }^{40}$ A. B. Meyer, ${ }^{40}$ G. Mittag, ${ }^{40}$ J. Mnich, ${ }^{40}$ A. Mussgiller ${ }^{40}$ E. Ntomari, ${ }^{40}$ D. Pitzl,${ }^{40}$ A. Raspereza, ${ }^{40}$ M. Savitskyi, ${ }^{40}$ P. Saxena, ${ }^{40}$ R. Shevchenko, ${ }^{40}$ N. Stefaniuk, ${ }^{40}$ G. P. Van Onsem, ${ }^{40}$ R. Walsh, ${ }^{40}$ Y. Wen, ${ }^{40}$ 
K. Wichmann, ${ }^{40}$ C. Wissing, ${ }^{40}$ O. Zenaiev,${ }^{40}$ R. Aggleton, ${ }^{41}$ S. Bein, ${ }^{41}$ V. Blobel, ${ }^{41}$ M. Centis Vignali, ${ }^{41}$ T. Dreyer, ${ }^{41}$ E. Garutti, ${ }^{41}$ D. Gonzalez, ${ }^{41}$ J. Haller, ${ }^{41}$ A. Hinzmann ${ }^{41}$ M. Hoffmann, ${ }^{41}$ A. Karavdina, ${ }^{41}$ R. Klanner ${ }^{41}$ R. Kogler ${ }^{41}$ N. Kovalchuk, ${ }^{41}$ S. Kurz ${ }^{41}$ T. Lapsien, ${ }^{41}$ D. Marconi, ${ }^{41}$ M. Meyer ${ }^{41}$ M. Niedziela, ${ }^{41}$ D. Nowatschin, ${ }^{41}$ F. Pantaleo, ${ }^{41, q}$ T. Peiffer, ${ }^{41}$ A. Perieanu, ${ }^{41}$ C. Scharf, ${ }^{41}$ P. Schleper, ${ }^{41}$ A. Schmidt, ${ }^{41}$ S. Schumann, ${ }^{41}$ J. Schwandt, ${ }^{41}$ J. Sonneveld, ${ }^{41}$ H. Stadie ${ }^{41}$ G. Steinbrück, ${ }^{41}$ F. M. Stober, ${ }^{41}$ M. Stöver ${ }^{41}$ H. Tholen, ${ }^{41}$ D. Troendle,${ }^{41}$ E. Usai, ${ }^{41}$ A. Vanhoefer, ${ }^{41}$ B. Vormwald ${ }^{41}$ M. Akbiyik, ${ }^{42}$ C. Barth,${ }^{42}$ M. Baselga, ${ }^{42}$ S. Baur, ${ }^{42}$ E. Butz, ${ }^{42}$ R. Caspart,${ }^{42}$ T. Chwalek, ${ }^{42}$ F. Colombo, ${ }^{42}$ W. De Boer, ${ }^{42}$ A. Dierlamm, ${ }^{42}$ N. Faltermann,${ }^{42}$ B. Freund,${ }^{42}$ R. Friese,${ }^{42}$ M. Giffels ${ }^{42}$ M. A. Harrendorf,${ }^{42}$ F. Hartmann, ${ }^{42, q}$ S. M. Heindl, ${ }^{42}$ U. Husemann, ${ }^{42}$ F. Kassel, ${ }^{42, q}$ S. Kudella, ${ }^{42}$ H. Mildner, ${ }^{42}$ M. U. Mozer, ${ }^{42}$ Th. Müller, ${ }^{42}$ M. Plagge, ${ }^{42}$ G. Quast, ${ }^{42}$ K. Rabbertz, ${ }^{42}$ M. Schröder, ${ }^{42}$ I. Shvetsov, ${ }^{42}$ G. Sieber, ${ }^{42}$ H. J. Simonis, ${ }^{42}$ R. Ulrich,${ }^{42}$ S. Wayand, ${ }^{42}$ M. Weber,${ }^{42}$ T. Weiler, ${ }^{42}$ S. Williamson, ${ }^{42}$ C. Wöhrmann, ${ }^{42}$ R. Wolf, ${ }^{42}$ G. Anagnostou, ${ }^{43}$ G. Daskalakis, ${ }^{43}$ T. Geralis, ${ }^{43}$ A. Kyriakis, ${ }^{43}$ D. Loukas, ${ }^{43}$ I. Topsis-Giotis, ${ }^{43}$ G. Karathanasis, ${ }^{44}$ S. Kesisoglou, ${ }^{44}$ A. Panagiotou, ${ }^{44}$ N. Saoulidou ${ }^{44}$ K. Kousouris ${ }^{45}$ I. Evangelou, ${ }^{46}$ C. Foudas, ${ }^{46}$ P. Gianneios, ${ }^{46}$ P. Katsoulis ${ }^{46}$ P. Kokkas, ${ }^{46}$ S. Mallios, ${ }^{46}$ N. Manthos, ${ }^{46}$ I. Papadopoulos ${ }^{46}$ E. Paradas ${ }^{46}$ J. Strologas, ${ }^{46}$ F. A. Triantis ${ }^{46}$ D. Tsitsonis, ${ }^{46}$ M. Csanad, ${ }^{47}$ N. Filipovic, ${ }^{47}$ G. Pasztor ${ }^{47}$ O. Surányi, ${ }^{47}$ G. I. Veres, ${ }^{47, u}$ G. Bencze, ${ }^{48}$ C. Hajdu, ${ }^{48}$ D. Horvath, ${ }^{48, v}$ Á. Hunyadi, ${ }^{48}$ F. Sikler, ${ }^{48}$ V. Veszpremi, ${ }^{48}$ N. Beni, ${ }^{49}$ S. Czellar, ${ }^{49}$ J. Karancsi ${ }^{49, w}$ A. Makovec, ${ }^{49}$ J. Molnar, ${ }^{49}$ Z. Szillasi, ${ }^{49}$ M. Bartók, ${ }^{50, u}$ P. Raics, ${ }^{50}$ Z. L. Trocsanyi,${ }^{50}$ B. Ujvari, ${ }^{50}$ S. Choudhury, ${ }^{51}$ J. R. Komaragiri, ${ }^{51}$ S. Bahinipati, ${ }^{52, x}$ S. Bhowmik, ${ }^{52}$ P. Mal ${ }^{52}$ K. Mandal, ${ }^{52}$ A. Nayak, ${ }^{52, y}$ D. K. Sahoo, ${ }^{52, x}$ N. Sahoo, ${ }^{52}$ S. K. Swain, ${ }^{52}$ S. Bansal,${ }^{53}$ S. B. Beri, ${ }^{53}$ V. Bhatnagar, ${ }^{53}$ R. Chawla,${ }^{53}$ N. Dhingra, ${ }^{53}$ A. Kaur,${ }^{53}$ M. Kaur,${ }^{53}$ S. Kaur ${ }^{53}$ R. Kumar, ${ }^{53}$ P. Kumari, ${ }^{53}$ A. Mehta, ${ }^{53}$ J. B. Singh,${ }^{53}$ G. Walia, ${ }^{53}$ Ashok Kumar, ${ }^{54}$ Aashaq Shah, ${ }^{54}$ A. Bhardwaj, ${ }^{54}$ S. Chauhan, ${ }^{54}$ B. C. Choudhary, ${ }^{54}$ R. B. Garg, ${ }^{54}$ S. Keshri, ${ }^{54}$ A. Kumar, ${ }^{54}$ S. Malhotra,${ }^{54}$ M. Naimuddin, ${ }^{54}$ K. Ranjan, ${ }^{54}$ R. Sharma ${ }^{54}$ R. Bhardwaj, ${ }^{55}$ R. Bhattacharya, ${ }^{55}$ S. Bhattacharya, ${ }^{55}$ U. Bhawandeep, ${ }^{55}$ S. Dey, ${ }^{55}$ S. Dutt,${ }^{55}$ S. Dutta ${ }^{55}$ S. Ghosh, ${ }^{55}$ N. Majumdar, ${ }^{55}$ A. Modak, ${ }^{55}$ K. Mondal, ${ }^{55}$ S. Mukhopadhyay, ${ }^{55}$ S. Nandan, ${ }^{55}$ A. Purohit,${ }^{55}$ A. Roy, ${ }^{55}$ S. Roy Chowdhury, ${ }^{55}$ S. Sarkar ${ }^{55}$ M. Sharan, ${ }^{55}$ S. Thakur, ${ }^{55}$ P. K. Behera ${ }^{56}$ R. Chudasama, ${ }^{57}$ D. Dutta, ${ }^{57}$ V. Jha ${ }^{57}$ V. Kumar, ${ }^{57}$ A. K. Mohanty, ${ }^{57, q}$ P. K. Netrakanti, ${ }^{57}$ L. M. Pant, ${ }^{57}$ P. Shukla, ${ }^{57}$ A. Topkar, ${ }^{57}$ T. Aziz, ${ }^{58}$ S. Dugad ${ }^{58}$ B. Mahakud ${ }^{58}$ S. Mitra,${ }^{58}$ G. B. Mohanty, ${ }^{58}$ N. Sur,${ }^{58}$ B. Sutar, ${ }^{58}$ S. Banerjee,${ }^{59}$ S. Bhattacharya,${ }^{59}$ S. Chatterjee,${ }^{59}$ P. Das,${ }^{59}$ M. Guchait, ${ }^{59}$ Sa. Jain, ${ }^{59}$ S. Kumar, ${ }^{59}$ M. Maity, ${ }^{59, z}$ G. Majumder,${ }^{59}$ K. Mazumdar, ${ }^{59}$ T. Sarkar, ${ }^{59, z}$ N. Wickramage, ${ }^{59, \text { aa }}$ S. Chauhan, ${ }^{60}$ S. Dube ${ }^{60}$ V. Hegde, ${ }^{60}$ A. Kapoor, ${ }^{60}$ K. Kothekar, ${ }^{60}$ S. Pandey,${ }^{60}$ A. Rane, ${ }^{60}$ S. Sharma, ${ }^{60}$ S. Chenarani, ${ }^{61, b b}$ E. Eskandari Tadavani, ${ }^{61}$ S. M. Etesami, ${ }^{61, b b}$ M. Khakzad, ${ }^{61}$ M. Mohammadi Najafabadi, ${ }^{61}$ M. Naseri, ${ }^{61}$

S. Paktinat Mehdiabadi, ${ }^{61, c c}$ F. Rezaei Hosseinabadi, ${ }^{61}$ B. Safarzadeh, ${ }^{61, d d}$ M. Zeinali ${ }^{61}$ M. Felcini, ${ }^{62}$ M. Grunewald ${ }^{62}$ M. Abbrescia ${ }^{63 a, 63 b}$ C. Calabria, ${ }^{63 a, 63 b}$ A. Colaleo, ${ }^{63 a}$ D. Creanza, ${ }^{63 a, 63 c}$ L. Cristella, ${ }^{63 a, 63 b}$ N. De Filippis, ${ }^{63 a, 63 c}$ M. De Palma, ${ }^{63 a, 63 b}$ F. Errico, ${ }^{63 a, 63 b}$ L. Fiore, ${ }^{63 a}$ G. Iaselli, ${ }^{63 a, 63 c}$ S. Lezki, ${ }^{63 a, 63 b}$ G. Maggi, ${ }^{63 a, 63 c}$ M. Maggi, ${ }^{63 a}$ G. Miniello, ${ }^{63 a, 63 b}$ S. My ${ }^{63 a, 63 b}$ S. Nuzzo, ${ }^{63 a, 63 b}$ A. Pompili, ${ }^{63 a, 63 b}$ G. Pugliese,${ }^{63 a, 63 c}$ R. Radogna,${ }^{63 a}$ A. Ranieri, ${ }^{63 a}$ G. Selvaggi, ${ }^{63 a, 63 b}$ A. Sharma, ${ }^{63 a}$ L. Silvestris, ${ }^{63 a, q}$ R. Venditti, ${ }^{63 a}$ P. Verwilligen, ${ }^{63 a}$ G. Abbiendi, ${ }^{64 a}$ C. Battilana, ${ }^{64 a, 64 b}$ D. Bonacorsi, ${ }^{64 a, 64 b}$ L. Borgonovi, ${ }^{64 a, 64 b}$ S. Braibant-Giacomelli, ${ }^{64 a, 64 b}$ R. Campanini, ${ }^{64 a, 64 b}$ P. Capiluppi, ${ }^{64 a, 64 b}$ A. Castro, ${ }^{64 a 64 b}$ F. R. Cavallo, ${ }^{64 a}$ S. S. Chhibra ${ }^{64 a}$ G. Codispoti ${ }^{64 a, 64 b}$ M. Cuffiani,${ }^{64 a, 64 b}$ G. M. Dallavalle ${ }^{64 a}$ F. Fabbri, ${ }^{64 a}$ A. Fanfani, ${ }^{64 a, 64 b}$ D. Fasanella, ${ }^{64 a, 64 b}$ P. Giacomelli, ${ }^{64 a}$ C. Grandi, ${ }^{64 a}$ L. Guiducci, ${ }^{64 a, 64 b}$ S. Marcellini, ${ }^{64 a}$ G. Masetti, ${ }^{64 a}$ A. Montanari, ${ }^{64 a}$ F. L. Navarria, ${ }^{64 a, 64 b}$ A. Perrotta, ${ }^{64 a}$ A. M. Rossi,${ }^{64 a, 64 b}$ T. Rovelli, ${ }^{64 a, 64 b}$ G. P. Siroli,${ }^{64 a, 64 b}$ N. Tosi, ${ }^{64 a}$ S. Albergo ${ }^{65 a, 65 b}$ S. Costa, ${ }^{65 a, 65 b}$ A. Di Mattia, ${ }^{65 a}$ F. Giordano, ${ }^{65 a, 65 b}$ R. Potenza, ${ }^{65 a, 65 b}$ A. Tricomi, ${ }^{65 a, 65 b}$ C. Tuve, ${ }^{65 a, 65 b}$ G. Barbagli, ${ }^{66 \mathrm{a}}$ K. Chatterjee ${ }^{66 a, 66 \mathrm{~b}}$ V. Ciulli, ${ }^{66 a, 66 \mathrm{~b}}$ C. Civinini, ${ }^{66 \mathrm{a}}$ R. D’Alessandro, ${ }^{66 a, 66 \mathrm{~b}}$ E. Focardi, ${ }^{66 a, 66 \mathrm{~b}}$ P. Lenzi, ${ }^{66 a, 66 \mathrm{~b}}$ M. Meschini, ${ }^{66 a}$ S. Paoletti, ${ }^{66 a}$ L. Russo, ${ }^{66 a, e e}$ G. Sguazzoni, ${ }^{66 a}$ D. Strom, ${ }^{66 a}$ L. Viliani, ${ }^{66 a}$ L. Benussi, ${ }^{67}$ S. Bianco, ${ }^{67}$ F. Fabbri, ${ }^{67}$ D. Piccolo, ${ }^{67}$ F. Primavera, ${ }^{67, q}$ V. Calvelli, ${ }^{68 a, 68 b}$ F. Ferro, ${ }^{68 a}$ F. Ravera, ${ }^{68 a, 68 b}$ E. Robutti, ${ }^{68 a}$ S. Tosi, ${ }^{68 a, 68 b}$ A. Benaglia, ${ }^{69 \mathrm{a}}$ A. Beschi, ${ }^{69 \mathrm{~b}}$ L. Brianza, ${ }^{69 \mathrm{a}, 69 \mathrm{~b}}$ F. Brivio, ${ }^{69 \mathrm{a}, 69 \mathrm{~b}}$ V. Ciriolo, ${ }^{69 \mathrm{a}, 69 \mathrm{~b}, \mathrm{q}}$ M. E. Dinardo,${ }^{69 \mathrm{a}, 69 \mathrm{~b}}$ S. Fiorendi, ${ }^{69 \mathrm{a}, 69 \mathrm{~b}}$ S. Gennai, ${ }^{69 a}$ A. Ghezzi, ${ }^{69 a, 69 b}$ P. Govoni ${ }^{69 a, 69 b}$ M. Malberti ${ }^{69 a, 69 b}$ S. Malvezzii, ${ }^{69 a}$ R. A. Manzoni, ${ }^{69 a, 69 b}$ D. Menasce, ${ }^{69 a}$ L. Moroni, ${ }^{69 a}$ M. Paganoni, ${ }^{69 a, 69 b}$ K. Pauwels, ${ }^{69 a, 69 b}$ D. Pedrini, ${ }^{69 a}$ S. Pigazzini, ${ }^{69 a, 69 b, f f}$ S. Ragazzi, ${ }^{69 a, 69 b}$

T. Tabarelli de Fatis ${ }^{69 a, 69 b}$ S. Buontempo, ${ }^{70 a}$ N. Cavallo, ${ }^{70 a, 70 c}$ S. Di Guida ${ }^{70 a, 70 d, q}$ F. Fabozzi, ${ }^{70 a, 70 c}$ F. Fienga, ${ }^{70 a, 70 b}$ A. O. M. Iorio, ${ }^{70 a, 70 b}$ W. A. Khan, ${ }^{70 a}$ L. Lista, ${ }^{70 a}$ S. Meola,${ }^{70 a, 70 d, q}$ P. Paolucci,${ }^{70 a, q}$ C. Sciacca, ${ }^{70 a, 70 b}$ F. Thyssen, ${ }^{70 a}$ P. Azzi, ${ }^{71 a}$ N. Bacchetta, ${ }^{71 a}$ L. Benato, ${ }^{71 a, 71 b}$ D. Bisello, ${ }^{71 a, 71 b}$ A. Boletti, ${ }^{71 a, 71 b}$ R. Carlin, ${ }^{71 a, 71 b}$ A. Carvalho Antunes De Oliveira, ${ }^{71 a, 71 b}$ P. Checchia, ${ }^{71 \mathrm{a}}$ P. De Castro Manzano, ${ }^{71 \mathrm{a}}$ T. Dorigo, ${ }^{71 \mathrm{a}}$ U. Dosselli, ${ }^{71 \mathrm{a}}$ F. Gasparini, ${ }^{71 \mathrm{a}, 71 \mathrm{~b}}$ U. Gasparini, ${ }^{71 \mathrm{a}, 71 \mathrm{~b}}$ A. Gozzelino, ${ }^{71 \mathrm{a}}$ S. Lacaprara, ${ }^{71 \mathrm{a}}$ M. Margoni, ${ }^{71 \mathrm{a}, 71 \mathrm{~b}}$ A. T. Meneguzzo, ${ }^{71 \mathrm{a}, 71 \mathrm{~b}}$ N. Pozzobon, ${ }^{71 \mathrm{a}, 71 \mathrm{~b}}$ P. Ronchese, ${ }^{71 \mathrm{a}, 71 \mathrm{~b}}$ R. Rossin, ${ }^{71 \mathrm{a}, 71 \mathrm{~b}}$ F. Simonetto, ${ }^{71 \mathrm{a}, 71 \mathrm{~b}}$ E. Torassa, ${ }^{71 \mathrm{a}}$ M. Zanetti, ${ }^{71 \mathrm{a}, 71 \mathrm{~b}}$ P. Zotto, ${ }^{71,71 \mathrm{~b}}$ G. Zumerle, ${ }^{71 \mathrm{a}, 7 \mathrm{~b}}$ A. Braghieri, ${ }^{72 \mathrm{a}}$ 
A. Magnani, ${ }^{72 a}$ P. Montagna, ${ }^{72 a, 72 b}$ S. P. Ratti, ${ }^{72 a, 72 b}$ V. Re,${ }^{72 a}$ M. Ressegotti, ${ }^{72 a, 72 b}$ C. Riccardi ${ }^{72 a, 72 b}$ P. Salvini, ${ }^{72 a}$ I. Vai, ${ }^{72 a, 72 b}$ P. Vitulo, ${ }^{72 a, 72 b}$ L. Alunni Solestizi,${ }^{73 a, 73 b}$ M. Biasini, ${ }^{73 a, 73 b}$ G. M. Bilei, ${ }^{73 a}$ C. Cecchi, ${ }^{73 a, 73 b}$ D. Ciangottini, ${ }^{73 a, 73 b}$ L. Fanò,${ }^{73 a, 73 b}$ R. Leonardi, ${ }^{73 a, 73 b}$ E. Manoni, ${ }^{73 a}$ G. Mantovani, ${ }^{73 a, 73 b}$ V. Mariani, ${ }^{73 a, 73 b}$ M. Menichelli, ${ }^{73 a}$ A. Rossi, ${ }^{73 a, 73 b}$ A. Santocchia, ${ }^{73 a, 73 b}$ D. Spiga, ${ }^{73 a}$ K. Androsov,${ }^{74 a}$ P. Azzurri, ${ }^{74 a, q}$ G. Bagliesi,${ }^{74 a}$ T. Boccali, ${ }^{74 a}$ L. Borrello, ${ }^{74 a}$ R. Castaldi, ${ }^{74 a}$ M. A. Ciocci, ${ }^{74 a, 74 b}$ R. Dell'Orso, ${ }^{74 a}$ G. Fedi, ${ }^{74 a}$ L. Giannini, ${ }^{74 a, 74 c}$ A. Giassi, ${ }^{74 a}$ M. T. Grippo, ${ }^{74 a, e e}$ F. Ligabue,${ }^{74 a, 74 c}$ T. Lomtadze, ${ }^{74 a}$ E. Manca, ${ }^{74 a, 74 c}$ G. Mandorli, ${ }^{74 a, 74 c}$ A. Messineo ${ }^{74 a, 74 b}$ F. Palla ${ }^{74 a}$ A. Rizzi, ${ }^{74 a, 74 b}$ A. Savoy-Navarro, ${ }^{74 a, g g}$ P. Spagnolo, ${ }^{74 a}$ R. Tenchini ${ }^{74 a}$ G. Tonelli, ${ }^{74 a, 74 b}$ A. Venturi, ${ }^{74 a}$ P. G. Verdini, ${ }^{74 a}$ L. Barone, ${ }^{75 a, 75 b}$ F. Cavallari, ${ }^{75 a}$ M. Cipriani, ${ }^{75 a, 75 b}$ N. Daci, ${ }^{75 a}$ D. Del Re ${ }^{75 a, 75 b, q}$ E. Di Marco, ${ }^{75 a, 75 b}$ M. Diemoz, ${ }^{75 a}$ S. Gelli, ${ }^{75 a, 75 b}$ E. Longo, ${ }^{75 a, 75 b}$ F. Margaroli, ${ }^{75 a, 75 b}$ B. Marzocchi, ${ }^{75 a, 75 b}$ P. Meridiani,${ }^{75 a}$ G. Organtini, ${ }^{75 a, 75 b}$ R. Paramatti, ${ }^{75 a, 75 b}$ F. Preiato, ${ }^{75 a, 75 b}$ S. Rahatlou, ${ }^{75 a, 75 b}$ C. Rovelli, ${ }^{75 a}$ F. Santanastasio, ${ }^{75 a, 75 b}$ N. Amapane, ${ }^{76 a, 76 b}$ R. Arcidiacono, ${ }^{76 a, 76 c}$ S. Argiro, ${ }^{76 a, 76 b}$ M. Arneodo, ${ }^{76 a, 76 c}$ N. Bartosik, ${ }^{76 a}$ R. Bellan, ${ }^{76 a, 76 b}$ C. Biino, ${ }^{76 a}$ N. Cartiglia, ${ }^{76 a}$ F. Cenna, ${ }^{76 a, 76 b}$ M. Costa,${ }^{76 a, 76 b}$ R. Covarelli, ${ }^{76 a, 76 b}$ A. Degano, ${ }^{76 a, 76 b}$ N. Demaria, ${ }^{76 a}$ B. Kiani, ${ }^{76 a, 76 b}$ C. Mariotti, ${ }^{76 a}$ S. Maselli, ${ }^{76 a}$ E. Migliore, ${ }^{76 a, 76 b}$ V. Monaco, ${ }^{76 a, 76 b}$ E. Monteil, ${ }^{76 a, 76 b}$ M. Monteno, ${ }^{76 a}$ M. M. Obertino, ${ }^{76 a, 76 b}$ L. Pacher, ${ }^{76 a, 76 b}$ N. Pastrone, ${ }^{76 a}$ M. Pelliccioni, ${ }^{76 a}$ G. L. Pinna Angioni, ${ }^{76 a, 76 b}$ A. Romero, ${ }^{76 a, 76 b}$ M. Ruspa, ${ }^{76 a, 76 c}$ R. Sacchi, ${ }^{76 a, 76 b}$ K. Shchelina, ${ }^{76 a, 76 b}$ V. Sola, ${ }^{76 a}$

A. Solano, ${ }^{76 a, 76 b}$ A. Staiano, ${ }^{76 a}$ P. Traczyk, ${ }^{76 a, 76 b}$ S. Belforte, ${ }^{77 a}$ M. Casarsa, ${ }^{77 a}$ F. Cossutti, ${ }^{77 a}$ G. Della Ricca, ${ }^{77 a, 77 b}$ A. Zanetti ${ }^{77 a}$ D. H. Kim, ${ }^{78}$ G. N. Kim, ${ }^{78}$ M. S. Kim,${ }^{78}$ J. Lee, ${ }^{78}$ S. Lee, ${ }^{78}$ S. W. Lee ${ }^{78}$ C. S. Moon ${ }^{78}$ Y. D. Oh, ${ }^{78}$ S. Sekmen, ${ }^{78}$ D. C. Son, ${ }^{78}$ Y. C. Yang, ${ }^{78}$ A. Lee,${ }^{79}$ H. Kim,${ }^{80}$ D. H. Moon,${ }^{80}$ G. Oh, ${ }^{80}$ J. A. Brochero Cifuentes, ${ }^{81}$ J. Goh, ${ }^{81}$ T. J. Kim, ${ }^{81}$ S. Cho, ${ }^{82}$ S. Choi ${ }^{82}$ Y. Go, ${ }^{82}$ D. Gyun, ${ }^{82}$ S. Ha, ${ }^{82}$ B. Hong, ${ }^{82}$ Y. Jo, ${ }^{82}$ Y. Kim,${ }^{82}$ K. Lee, ${ }^{82}$ K. S. Lee, ${ }^{82}$ S. Lee, ${ }^{82}$ J. Lim, ${ }^{82}$ S. K. Park ${ }^{82}$ Y. Roh, ${ }^{82}$ J. Almond, ${ }^{83}$ J. Kim, ${ }^{83}$ J. S. Kim, ${ }^{83}$ H. Lee, ${ }^{83}$ K. Lee, ${ }^{83}$ K. Nam, ${ }^{83}$ S. B. Oh, ${ }^{83}$ B. C. Radburn-Smith, ${ }^{83}$ S. h. Seo, ${ }^{83}$ U. K. Yang, ${ }^{83}$ H. D. Yoo, ${ }^{83}$ G. B. Yu,${ }^{83}$ H. Kim, ${ }^{84}$ J. H. Kim, ${ }^{84}$ J. S. H. Lee, ${ }^{84}$ I. C. Park,${ }^{84}$ Y. Choi, ${ }^{85}$ C. Hwang, ${ }^{85}$ J. Lee, ${ }^{85}$ I. Yu, ${ }^{85}$ V. Dudenas, ${ }^{86}$ A. Juodagalvis, ${ }^{86}$ J. Vaitkus, ${ }^{86}$ I. Ahmed, ${ }^{87}$ Z. A. Ibrahim, ${ }^{87}$ M. A. B. Md Ali, ${ }^{87, \text { hh }}$ F. Mohamad Idris, ${ }^{87, i i}$ W. A. T. Wan Abdullah, ${ }^{87}$ M. N. Yusli, ${ }^{87}$ Z. Zolkapli, ${ }^{87}$ R. Reyes-Almanza ${ }^{88}$ G. Ramirez-Sanchez, ${ }^{88}$ M. C. Duran-Osuna, ${ }^{88}$ H. Castilla-Valdez ${ }^{88}$ E. De La Cruz-Burelo, ${ }^{88}$ I. Heredia-De La Cruz, ${ }^{88, j j}$ R. I. Rabadan-Trejo ${ }^{88}$ R. Lopez-Fernandez, ${ }^{88}$ J. Mejia Guisao, ${ }^{88}$ A. Sanchez-Hernandez, ${ }^{88}$ S. Carrillo Moreno, ${ }^{89}$ C. Oropeza Barrera, ${ }^{89}$ F. Vazquez Valencia, ${ }^{89}$ J. Eysermans,${ }^{90}$ I. Pedraza,${ }^{90}$ H. A. Salazar Ibarguen,${ }^{90}$ C. Uribe Estrada,${ }^{90}$ A. Morelos Pineda, ${ }^{91}$ D. Krofcheck, ${ }^{92}$ P. H. Butler, ${ }^{93}$ A. Ahmad, ${ }^{94}$ M. Ahmad, ${ }^{94}$ Q. Hassan,${ }^{94}$ H. R. Hoorani, ${ }^{94}$ A. Saddique,${ }^{94}$ M. A. Shah, ${ }^{94}$ M. Shoaib, ${ }^{94}$ M. Waqas, ${ }^{94}$ H. Bialkowska, ${ }^{95}$ M. Bluj,${ }^{95}$ B. Boimska, ${ }^{95}$ T. Frueboes,${ }^{95}$ M. Górski, ${ }^{95}$ M. Kazana, ${ }^{95}$ K. Nawrocki, ${ }^{95}$ M. Szleper, ${ }^{95}$ P. Zalewski,${ }^{95}$ K. Bunkowski, ${ }^{96}$ A. Byszuk, ${ }^{96, k k}$ K. Doroba ${ }^{96}$ A. Kalinowski,${ }^{96}$ M. Konecki, ${ }^{96}$ J. Krolikowski, ${ }^{96}$ M. Misiura, ${ }^{96}$ M. Olszewski, ${ }^{96}$ A. Pyskir, ${ }^{96}$ M. Walczak,${ }^{96}$ P. Bargassa, ${ }^{97}$ C. Beirão Da Cruz E Silva ${ }^{97}$ A. Di Francesco, ${ }^{97}$ P. Faccioli, ${ }^{97}$ B. Galinhas, ${ }^{97}$ M. Gallinaro, ${ }^{97}$ J. Hollar, ${ }^{97}$ N. Leonardo, ${ }^{97}$ L. Lloret Iglesias, ${ }^{97}$ M. V. Nemallapudi, ${ }^{97}$ J. Seixas, ${ }^{97}$ G. Strong, ${ }^{97}$ O. Toldaiev,${ }^{97}$ D. Vadruccio, ${ }^{97}$ J. Varela, ${ }^{97}$ S. Afanasiev,${ }^{98}$ P. Bunin, ${ }^{98}$ M. Gavrilenko, ${ }^{98}$ I. Golutvin, ${ }^{98}$ I. Gorbunov,${ }^{98}$ A. Kamenev, ${ }^{98}$ V. Karjavin, ${ }^{98}$ A. Lanev,${ }^{98}$ A. Malakhov, ${ }^{98}$ V. Matveev, ${ }^{98,11, m m}$ V. Palichik, ${ }^{98}$ V. Perelygin, ${ }^{98}$ S. Shmatov ${ }^{98}$ S. Shulha,${ }^{98}$ N. Skatchkov, ${ }^{98}$ V. Smirnov, ${ }^{98}$ N. Voytishin, ${ }^{98}$ A. Zarubin,${ }^{98}$ Y. Ivanov, ${ }^{99}$ V. Kim, ${ }^{99, \text { nn }}$ E. Kuznetsova,${ }^{99,00}$ P. Levchenko, ${ }^{99}$ V. Murzin,${ }^{99}$ V. Oreshkin, ${ }^{99}$ I. Smirnov, ${ }^{99}$ D. Sosnov, ${ }^{99}$ V. Sulimov, ${ }^{99}$ L. Uvarov, ${ }^{99}$ S. Vavilov, ${ }^{99}$ A. Vorobyev, ${ }^{99}$ Yu. Andreev, ${ }^{100}$ A. Dermenev, ${ }^{100}$ S. Gninenko, ${ }^{100}$ N. Golubev, ${ }^{100}$ A. Karneyeu, ${ }^{100}$ M. Kirsanov, ${ }^{100}$ N. Krasnikov, ${ }^{100}$ A. Pashenkov, ${ }^{100}$ D. Tlisov,${ }^{100}$ A. Toropin, ${ }^{100}$ V. Epshteyn,${ }^{101}$ V. Gavrilov, ${ }^{101}$ N. Lychkovskaya, ${ }^{101}$ V. Popov, ${ }^{101}$ I. Pozdnyakov, ${ }^{101}$ G. Safronov ${ }^{101}$ A. Spiridonov, ${ }^{101}$ A. Stepennov, ${ }^{101}$ M. Toms, ${ }^{101}$ E. Vlasov, ${ }^{101}$ A. Zhokin, ${ }^{101}$ T. Aushev, ${ }^{102}$ A. Bylinkin, ${ }^{102, m m}$ R. Chistov, ${ }^{103, p p}$ M. Danilov, ${ }^{103, p p}$ P. Parygin, ${ }^{103}$ D. Philippov, ${ }^{103}$ S. Polikarpov, ${ }^{103}$ E. Tarkovskii, ${ }^{103}$ V. Andreev, ${ }^{104}$ M. Azarkin, ${ }^{104, m m}$ I. Dremin, ${ }^{104, m m}$ M. Kirakosyan, ${ }^{104, m m}$ A. Terkulov, ${ }^{104}$ A. Baskakov, ${ }^{105}$ A. Belyaev, ${ }^{105}$ E. Boos, ${ }^{105}$ L. Dudko, ${ }^{105}$ A. Ershov, ${ }^{105}$ A. Gribushin, ${ }^{105}$ O. Kodolova, ${ }^{105}$ V. Korotkikh, ${ }^{105}$ I. Lokhtin, ${ }^{105}$ I. Miagkov, ${ }^{105}$ S. Obraztsov, ${ }^{105}$ S. Petrushanko, ${ }^{105}$ V. Savrin, ${ }^{105}$ A. Snigirev, ${ }^{105}$ I. Vardanyan, ${ }^{105}$ V. Blinov, ${ }^{106, q q}$ Y. Skovpen, ${ }^{106, q q}$ D. Shtol, ${ }^{106, q q}$ I. Azhgirey, ${ }^{107}$ I. Bayshev, ${ }^{107}$ S. Bitioukov, ${ }^{107}$ D. Elumakhov, ${ }^{107}$ A. Godizov, ${ }^{107}$ V. Kachanov, ${ }^{107}$ A. Kalinin, ${ }^{107}$ D. Konstantinov, ${ }^{107}$ P. Mandrik, ${ }^{107}$ V. Petrov, ${ }^{107}$ R. Ryutin, ${ }^{107}$ A. Sobol, ${ }^{107}$ S. Troshin, ${ }^{107}$ N. Tyurin, ${ }^{107}$ A. Uzunian, ${ }^{107}$ A. Volkov, ${ }^{107}$ P. Adzic, ${ }^{108, r r}$ P. Cirkovic, ${ }^{108}$ D. Devetak, ${ }^{108}$ M. Dordevic, ${ }^{108}$ J. Milosevic, ${ }^{108}$ V. Rekovic, ${ }^{108}$ J. Alcaraz Maestre, ${ }^{109}$ I. Bachiller, ${ }^{109}$ M. Barrio Luna, ${ }^{109}$ M. Cerrada,${ }^{109}$ N. Colino, ${ }^{109}$ B. De La Cruz, ${ }^{109}$ A. Delgado Peris, ${ }^{109}$ C. Fernandez Bedoya, ${ }^{109}$ J. P. Fernández Ramos, ${ }^{109}$ J. Flix, ${ }^{109}$ M. C. Fouz, ${ }^{109}$ O. Gonzalez Lopez, ${ }^{109}$ S. Goy Lopez, ${ }^{109}$ J. M. Hernandez, ${ }^{109}$ M. I. Josa, ${ }^{109}$ D. Moran, ${ }^{109}$ A. Pérez-Calero Yzquierdo, ${ }^{109}$ J. Puerta Pelayo,${ }^{109}$ A. Quintario Olmeda,${ }^{109}$ I. Redondo ${ }^{109}$ L. Romero, ${ }^{109}$ M. S. Soares, ${ }^{109}$ A. Álvarez Fernández, ${ }^{109}$ C. Albajar, ${ }^{110}$ J. F. de Trocóniz, ${ }^{110}$ M. Missiroli, ${ }^{110}$ J. Cuevas, ${ }^{111}$ C. Erice, ${ }^{111}$ 
J. Fernandez Menendez, ${ }^{111}$ I. Gonzalez Caballero, ${ }^{111}$ J. R. González Fernández, ${ }^{111}$ E. Palencia Cortezon, ${ }^{111}$ S. Sanchez Cruz ${ }^{111}$ P. Vischia, ${ }^{111}$ J. M. Vizan Garcia, ${ }^{111}$ I. J. Cabrillo, ${ }^{112}$ A. Calderon, ${ }^{112}$ B. Chazin Quero, ${ }^{112}$ E. Curras, ${ }^{112}$ J. Duarte Campderros, ${ }^{112}$ M. Fernandez, ${ }^{112}$ J. Garcia-Ferrero, ${ }^{112}$ G. Gomez, ${ }^{112}$ A. Lopez Virto, ${ }^{112}$ J. Marco, ${ }^{112}$ C. Martinez Rivero, ${ }^{112}$ P. Martinez Ruiz del Arbol, ${ }^{112}$ F. Matorras, ${ }^{112}$ J. Piedra Gomez, ${ }^{112}$ T. Rodrigo, ${ }^{112}$ A. Ruiz-Jimeno, ${ }^{112}$ L. Scodellaro, ${ }^{112}$ N. Trevisani, ${ }^{112}$ I. Vila, ${ }^{112}$ R. Vilar Cortabitarte, ${ }^{112}$ D. Abbaneo, ${ }^{113}$ B. Akgun, ${ }^{113}$ E. Auffray, ${ }^{113}$ P. Baillon, ${ }^{113}$ A. H. Ball, ${ }^{113}$ D. Barney, ${ }^{113}$ J. Bendavid, ${ }^{113}$ M. Bianco, ${ }^{113}$ P. Bloch, ${ }^{113}$ A. Bocci, ${ }^{113}$ C. Botta, ${ }^{113}$ T. Camporesi, ${ }^{113}$ R. Castello, ${ }^{113}$ M. Cepeda, ${ }^{113}$ G. Cerminara, ${ }^{113}$ E. Chapon, ${ }^{113}$ Y. Chen, ${ }^{113}$ D. d'Enterria, ${ }^{113}$ A. Dabrowski, ${ }^{113}$ V. Daponte, ${ }^{113}$ A. David, ${ }^{113}$ M. De Gruttola, ${ }^{113}$ A. De Roeck, ${ }^{113}$ N. Deelen, ${ }^{113}$ M. Dobson, ${ }^{113}$ T. du Pree, ${ }^{113}$ M. Dünser, ${ }^{113}$ N. Dupont, ${ }^{113}$ A. Elliott-Peisert, ${ }^{113}$ P. Everaerts, ${ }^{113}$ F. Fallavollita, ${ }^{113}$ G. Franzoni, ${ }^{113}$ J. Fulcher, ${ }^{113}$ W. Funk, ${ }^{113}$ D. Gigi, ${ }^{113}$ A. Gilbert, ${ }^{113}$ K. Gill, ${ }^{113}$ F. Glege, ${ }^{113}$ D. Gulhan, ${ }^{113}$ P. Harris, ${ }^{113}$ J. Hegeman, ${ }^{113}$ V. Innocente, ${ }^{113}$ A. Jafari, ${ }^{113}$ P. Janot, ${ }^{113}$ O. Karacheban, ${ }^{11, t}$ J. Kieseler, ${ }^{113}$ V. Knünz, ${ }^{113}$ A. Kornmayer, ${ }^{113}$ M. J. Kortelainen, ${ }^{113}$ M. Krammer, ${ }^{113, b}$ C. Lange, ${ }^{113}$ P. Lecoq, ${ }^{113}$ C. Lourenço, ${ }^{113}$ M. T. Lucchini, ${ }^{113}$ L. Malgeri, ${ }^{113}$ M. Mannelli, ${ }^{113}$ A. Martelli, ${ }^{113}$ F. Meijers, ${ }^{113}$ J. A. Merlin ${ }^{113}$ S. Mersi, ${ }^{113}$ E. Meschi, ${ }^{113}$ P. Milenovic, ${ }^{113, s s}$ F. Moortgat, ${ }^{113}$ M. Mulders,${ }^{113}$ H. Neugebauer, ${ }^{113}$ J. Ngadiuba, ${ }^{113}$ S. Orfanelli, ${ }^{113}$ L. Orsini, ${ }^{113}$ L. Pape, ${ }^{113}$ E. Perez, ${ }^{113}$ M. Peruzzi, ${ }^{113}$ A. Petrilli, ${ }^{113}$ G. Petrucciani, ${ }^{113}$ A. Pfeiffer, ${ }^{113}$ M. Pierini, ${ }^{113}$ D. Rabady, ${ }^{113}$ A. Racz, ${ }^{113}$ T. Reis, ${ }^{113}$ G. Rolandi, ${ }^{113, t t}$ M. Rovere, ${ }^{113}$ H. Sakulin, ${ }^{113}$ C. Schäfer, ${ }^{113}$

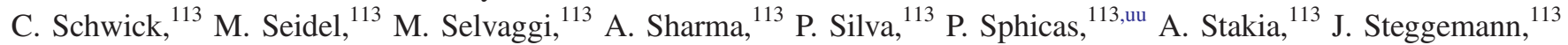
M. Stoye,${ }^{113}$ M. Tosi, ${ }^{113}$ D. Treille, ${ }^{113}$ A. Triossi, ${ }^{113}$ A. Tsirou, ${ }^{113}$ V. Veckalns,${ }^{11, v v}$ M. Verweij, ${ }^{113}$ W. D. Zeuner, ${ }^{113}$ W. Bertl, ${ }^{114, a}$ L. Caminada, ${ }^{114, w w}$ K. Deiters, ${ }^{114}$ W. Erdmann, ${ }^{114}$ R. Horisberger, ${ }^{114}$ Q. Ingram,${ }^{114}$ H. C. Kaestli, ${ }^{114}$ D. Kotlinski, ${ }^{114}$ U. Langenegger, ${ }^{114}$ T. Rohe, ${ }^{114}$ S. A. Wiederkehr, ${ }^{114}$ M. Backhaus, ${ }^{115}$ L. Bäni, ${ }^{115}$ P. Berger,${ }^{115}$ L. Bianchini, ${ }^{115}$ B. Casal, ${ }^{115}$ G. Dissertori, ${ }^{115}$ M. Dittmar, ${ }^{15}$ M. Donegà, ${ }^{115}$ C. Dorfer, ${ }^{115}$ C. Grab, ${ }^{115}$ C. Heidegger, ${ }^{115}$ D. Hits, ${ }^{115}$ J. Hoss, ${ }^{115}$ G. Kasieczka, ${ }^{115}$ T. Klijnsma, ${ }^{115}$ W. Lustermann, ${ }^{115}$ B. Mangano, ${ }^{115}$ M. Marionneau, ${ }^{115}$ M. T. Meinhard, ${ }^{115}$ D. Meister, ${ }^{115}$ F. Micheli, ${ }^{115}$ P. Musella, ${ }^{115}$ F. Nessi-Tedaldi, ${ }^{115}$ F. Pandolfi, ${ }^{115}$ J. Pata, ${ }^{115}$ F. Pauss, ${ }^{115}$ G. Perrin, ${ }^{115}$ L. Perrozzi, ${ }^{115}$ M. Quittnat, ${ }^{115}$ M. Reichmann, ${ }^{115}$ D. A. Sanz Becerra,${ }^{115}$ M. Schönenberger, ${ }^{115}$ L. Shchutska, ${ }^{115}$ V. R. Tavolaro, ${ }^{115}$ K. Theofilatos, ${ }^{115}$ M. L. Vesterbacka Olsson, ${ }^{115}$ R. Wallny, ${ }^{115}$ D. H. Zhu, ${ }^{115}$ T. K. Aarrestad, ${ }^{116}$ C. Amsler, ${ }^{116, x x}$ M. F. Canelli, ${ }^{116}$ A. De Cosa, ${ }^{116}$ R. Del Burgo,${ }^{116}$ S. Donato, ${ }^{116}$ C. Galloni, ${ }^{116}$ T. Hreus, ${ }^{116}$ B. Kilminster, ${ }^{116}$ D. Pinna, ${ }^{116}$ G. Rauco, ${ }^{116}$ P. Robmann, ${ }^{116}$ D. Salerno, ${ }^{116}$ K. Schweiger, ${ }^{116}$ C. Seitz, ${ }^{116}$ Y. Takahashi, ${ }^{116}$ A. Zucchetta, ${ }^{116}$ V. Candelise, ${ }^{117}$ Y. H. Chang, ${ }^{117}$ K. y. Cheng, ${ }^{117}$ T. H. Doan, ${ }^{117}$ Sh. Jain, ${ }^{117}$ R. Khurana, ${ }^{117}$ C. M. Kuo, ${ }^{117}$ W. Lin,${ }^{117}$ A. Pozdnyakov, ${ }^{117}$ S. S. Yu, ${ }^{117}$ Arun Kumar, ${ }^{118}$ P. Chang, ${ }^{118}$ Y. Chao, ${ }^{118}$ K. F. Chen ${ }^{118}$ P. H. Chen, ${ }^{118}$ F. Fiori, ${ }^{118}$ W.-S. Hou, ${ }^{118}$ Y. Hsiung, ${ }^{118}$ Y. F. Liu, ${ }^{118}$ R.-S. Lu, ${ }^{118}$ E. Paganis,${ }^{118}$ A. Psallidas, ${ }^{118}$ A. Steen, ${ }^{118}$ J. f. Tsai, ${ }^{118}$ B. Asavapibhop, ${ }^{119}$ K. Kovitanggoon, ${ }^{119}$ G. Singh, ${ }^{119}$ N. Srimanobhas, ${ }^{119}$ A. Bat, ${ }^{120}$ F. Boran, ${ }^{120}$ S. Cerci, ${ }^{120, y y}$ S. Damarseckin, ${ }^{120}$ Z. S. Demiroglu, ${ }^{120}$ C. Dozen, ${ }^{120}$ I. Dumanoglu, ${ }^{120}$ S. Girgis,${ }^{120}$ G. Gokbulut, ${ }^{120}$ Y. Guler, ${ }^{120}$ I. Hos, ${ }^{120, z z}$ E. E. Kangal, ${ }^{120, \text { aaa }}$ O. Kara, ${ }^{120}$ A. Kayis Topaksu, ${ }^{120}$ U. Kiminsu, ${ }^{120}$ M. Oglakci, ${ }^{120}$ G. Onengut, ${ }^{120, b b b}$ K. Ozdemir, ${ }^{120, c c c}$ D. Sunar Cerci, ${ }^{120, y y}$ B. Tali, ${ }^{120, y y}$ U. G. Tok, ${ }^{120}$ S. Turkcapar, ${ }^{120}$ I. S. Zorbakir, ${ }^{120}$ C. Zorbilmez, ${ }^{120}$ G. Karapinar, ${ }^{121, \text { ddd }}$ K. Ocalan, ${ }^{121, \text { eee }}$ M. Yalvac, ${ }^{121}$ M. Zeyrek, ${ }^{121}$ E. Gülmez, ${ }^{122}$ M. Kaya, ${ }^{122, f f f}$ O. Kaya, ${ }^{122, \text { ggg }}$ S. Tekten, ${ }^{122}$ E. A. Yetkin, ${ }^{122, \text { hhh }}$ M. N. Agaras, ${ }^{123}$ S. Atay, ${ }^{123}$ A. Cakir, ${ }^{123}$ K. Cankocak, ${ }^{123}$ I. Köseoglu, ${ }^{123}$ B. Grynyov, ${ }^{124}$ L. Levchuk, ${ }^{125}$ F. Ball, ${ }^{126}$ L. Beck, ${ }^{126}$ J. J. Brooke, ${ }^{126}$ D. Burns,${ }^{126}$ E. Clement,${ }^{126}$ D. Cussans, ${ }^{126}$ O. Davignon, ${ }^{126}$ H. Flacher, ${ }^{126}$ J. Goldstein, ${ }^{126}$ G. P. Heath, ${ }^{126}$ H. F. Heath, ${ }^{126}$ L. Kreczko, ${ }^{126}$ D. M. Newbold, ${ }^{126, \text { iii }}$ S. Paramesvaran, ${ }^{126}$ T. Sakuma, ${ }^{126}$

S. Seif El Nasr-storey, ${ }^{126}$ D. Smith, ${ }^{126}$ V. J. Smith, ${ }^{126}$ A. Belyaev, ${ }^{127, j j j}$ C. Brew, ${ }^{127}$ R. M. Brown, ${ }^{127}$ L. Calligaris, ${ }^{127}$ D. Cieri, ${ }^{127}$ D. J. A. Cockerill, ${ }^{127}$ J. A. Coughlan, ${ }^{127}$ K. Harder, ${ }^{127}$ S. Harper, ${ }^{127}$ J. Linacre, ${ }^{127}$ E. Olaiya,${ }^{127}$ D. Petyt, ${ }^{127}$ C. H. Shepherd-Themistocleous, ${ }^{127}$ A. Thea, ${ }^{127}$ I. R. Tomalin, ${ }^{127}$ T. Williams, ${ }^{127}$ G. Auzinger ${ }^{128}$ R. Bainbridge, ${ }^{128}$ J. Borg, ${ }^{128}$ S. Breeze, ${ }^{128}$ O. Buchmuller, ${ }^{128}$ A. Bundock, ${ }^{128}$ S. Casasso, ${ }^{128}$ M. Citron, ${ }^{128}$ D. Colling, ${ }^{128}$ L. Corpe, ${ }^{128}$ P. Dauncey, ${ }^{128}$ G. Davies, ${ }^{128}$ A. De Wit, ${ }^{128}$ M. Della Negra, ${ }^{128}$ R. Di Maria, ${ }^{128}$ A. Elwood, ${ }^{128}$ Y. Haddad, ${ }^{128}$ G. Hall, ${ }^{128}$ G. Iles, ${ }^{128}$ T. James, ${ }^{128}$ R. Lane, ${ }^{128}$ C. Laner, ${ }^{128}$ L. Lyons, ${ }^{128}$ A.-M. Magnan, ${ }^{128}$ S. Malik, ${ }^{128}$ L. Mastrolorenzo, ${ }^{128}$ T. Matsushita, ${ }^{128}$ J. Nash, ${ }^{128}$ A. Nikitenko, ${ }^{128,9}$ V. Palladino, ${ }^{128}$ M. Pesaresi ${ }^{128}$ D. M. Raymond, ${ }^{128}$ A. Richards, ${ }^{128}$ A. Rose,${ }^{128}$ E. Scott, ${ }^{128}$ C. Seez,${ }^{128}$ A. Shtipliyski, ${ }^{128}$ S. Summers, ${ }^{128}$ A. Tapper, ${ }^{128}$ K. Uchida,${ }^{128}$ M. Vazquez Acosta, ${ }^{128, k k k}$ T. Virdee, ${ }^{128, \mathrm{q}}$ N. Wardle, ${ }^{128}$ D. Winterbottom, ${ }^{128}$ J. Wright, ${ }^{128}$ S. C. Zenz, ${ }^{128}$ J. E. Cole, ${ }^{129}$ P. R. Hobson, ${ }^{129}$ A. Khan, ${ }^{129}$ P. Kyberd ${ }^{129}$ I. D. Reid, ${ }^{129}$ L. Teodorescu, ${ }^{129}$ S. Zahid, ${ }^{129}$ A. Borzou, ${ }^{130}$ K. Call,${ }^{130}$ J. Dittmann, ${ }^{130}$ K. Hatakeyama, ${ }^{130}$ H. Liu, ${ }^{130}$ N. Pastika, ${ }^{130}$ C. Smith, ${ }^{130}$ R. Bartek, ${ }^{131}$ A. Dominguez, ${ }^{131}$ A. Buccilli, ${ }^{132}$ S. I. Cooper, ${ }^{132}$ C. Henderson, ${ }^{132}$ P. Rumerio, ${ }^{132}$ C. West, ${ }^{132}$ D. Arcaro, ${ }^{133}$ A. Avetisyan, ${ }^{133}$ T. Bose, ${ }^{133}$ D. Gastler, ${ }^{133}$ D. Rankin, ${ }^{133}$ C. Richardson, ${ }^{133}$ 
J. Rohlf, ${ }^{133}$ L. Sulak, ${ }^{133}$ D. Zou, ${ }^{133}$ G. Benelli, ${ }^{134}$ D. Cutts ${ }^{134}$ A. Garabedian, ${ }^{134}$ M. Hadley, ${ }^{134}$ J. Hakala, ${ }^{134}$ U. Heintz, ${ }^{134}$ J. M. Hogan, ${ }^{134}$ K. H. M. Kwok, ${ }^{134}$ E. Laird, ${ }^{134}$ G. Landsberg, ${ }^{134}$ J. Lee, ${ }^{134}$ Z. Mao, ${ }^{134}$ M. Narain, ${ }^{134}$ J. Pazzini, ${ }^{134}$ S. Piperov, ${ }^{134}$ S. Sagir, ${ }^{134}$ R. Syarif, ${ }^{134}$ D. Yu, ${ }^{134}$ R. Band, ${ }^{135}$ C. Brainerd, ${ }^{135}$ D. Burns, ${ }^{135}$

M. Calderon De La Barca Sanchez, ${ }^{135}$ M. Chertok,${ }^{135}$ J. Conway, ${ }^{135}$ R. Conway, ${ }^{135}$ P. T. Cox, ${ }^{135}$ R. Erbacher, ${ }^{135}$ C. Flores, ${ }^{135}$ G. Funk, ${ }^{135}$ W. Ko, ${ }^{135}$ R. Lander, ${ }^{135}$ C. Mclean, ${ }^{135}$ M. Mulhearn, ${ }^{135}$ D. Pellett, ${ }^{135}$ J. Pilot, ${ }^{135}$ S. Shalhout, ${ }^{135}$ M. Shi, ${ }^{135}$ J. Smith, ${ }^{135}$ D. Stolp, ${ }^{135}$ K. Tos, ${ }^{135}$ M. Tripathi, ${ }^{135}$ Z. Wang, ${ }^{135}$ M. Bachtis,${ }^{136}$ C. Bravo, ${ }^{136}$ R. Cousins, ${ }^{136}$ A. Dasgupta, ${ }^{136}$ A. Florent, ${ }^{136}$ J. Hauser, ${ }^{136}$ M. Ignatenko, ${ }^{136}$ N. Mccoll, ${ }^{136}$ S. Regnard, ${ }^{136}$ D. Saltzberg, ${ }^{136}$ C. Schnaible, ${ }^{136}$ V. Valuev, ${ }^{136}$ E. Bouvier, ${ }^{137}$ K. Burt, ${ }^{137}$ R. Clare, ${ }^{137}$ J. Ellison, ${ }^{137}$ J. W. Gary, ${ }^{137}$ S. M. A. Ghiasi Shirazi, ${ }^{137}$ G. Hanson, ${ }^{137}$ J. Heilman, ${ }^{137}$ G. Karapostoli, ${ }^{137}$ E. Kennedy, ${ }^{137}$ F. Lacroix, ${ }^{137}$ O. R. Long, ${ }^{137}$ M. Olmedo Negrete, ${ }^{137}$ M. I. Paneva, ${ }^{137}$ W. Si, ${ }^{137}$ L. Wang, ${ }^{137}$ H. Wei, ${ }^{137}$ S. Wimpenny, ${ }^{137}$ B. R. Yates, ${ }^{137}$ J. G. Branson, ${ }^{138}$ S. Cittolin, ${ }^{138}$ M. Derdzinski, ${ }^{138}$ R. Gerosa, ${ }^{138}$ D. Gilbert, ${ }^{138}$ B. Hashemi, ${ }^{138}$ A. Holzner, ${ }^{138}$ D. Klein, ${ }^{138}$ G. Kole, ${ }^{138}$ V. Krutelyov, ${ }^{138}$ J. Letts, ${ }^{138}$ M. Masciovecchio, ${ }^{138}$ D. Olivito, ${ }^{138}$ S. Padhi, ${ }^{138}$ M. Pieri, ${ }^{138}$ M. Sani,${ }^{138}$ V. Sharma, ${ }^{138}$ M. Tadel, ${ }^{138}$ A. Vartak, ${ }^{138}$ S. Wasserbaech,${ }^{138,111}$ J. Wood, ${ }^{138}$ F. Würthwein, ${ }^{138}$ A. Yagil, ${ }^{138}$ G. Zevi Della Porta,${ }^{138}$ N. Amin, ${ }^{139}$ R. Bhandari, ${ }^{139}$ J. Bradmiller-Feld, ${ }^{139}$ C. Campagnari, ${ }^{139}$ A. Dishaw, ${ }^{139}$ V. Dutta, ${ }^{139}$ M. Franco Sevilla, ${ }^{139}$ L. Gouskos, ${ }^{139}$ R. Heller, ${ }^{139}$ J. Incandela, ${ }^{139}$ A. Ovcharova, ${ }^{139}$ H. Qu, ${ }^{139}$ J. Richman, ${ }^{139}$ D. Stuart, ${ }^{139}$ I. Suarez,${ }^{139}$ J. Yoo, ${ }^{139}$ D. Anderson, ${ }^{140}$ A. Bornheim, ${ }^{140}$ J. M. Lawhorn, ${ }^{140}$ H. B. Newman, ${ }^{140}$ T. Q. Nguyen, ${ }^{140}$ C. Pena,${ }^{140}$ M. Spiropulu, ${ }^{140}$ J. R. Vlimant ${ }^{140}$ S. Xie, ${ }^{140}$ Z. Zhang, ${ }^{140}$ R. Y. Zhu, ${ }^{140}$ M. B. Andrews, ${ }^{141}$ T. Ferguson, ${ }^{141}$ T. Mudholkar, ${ }^{141}$ M. Paulini, ${ }^{141}$ J. Russ, ${ }^{141}$ M. Sun,${ }^{141}$ H. Vogel, ${ }^{141}$ I. Vorobiev,${ }^{141}$ M. Weinberg, ${ }^{141}$ J. P. Cumalat, ${ }^{142}$ W. T. Ford, ${ }^{142}$ F. Jensen, ${ }^{142}$ A. Johnson, ${ }^{142}$ M. Krohn, ${ }^{142}$ S. Leontsinis, ${ }^{142}$ T. Mulholland, ${ }^{142}$ K. Stenson, ${ }^{142}$ S. R. Wagner, ${ }^{142}$ J. Alexander, ${ }^{143}$ J. Chaves,${ }^{143}$ J. Chu, ${ }^{143}$ S. Dittmer, ${ }^{143}$ K. Mcdermott, ${ }^{143}$ N. Mirman, ${ }^{143}$ J. R. Patterson, ${ }^{143}$ D. Quach, ${ }^{143}$ A. Rinkevicius, ${ }^{143}$ A. Ryd ${ }^{143}$ L. Skinnari, ${ }^{143}$ L. Soffi, ${ }^{143}$ S. M. Tan, ${ }^{143}$ Z. Tao, ${ }^{143}$ J. Thom, ${ }^{143}$ J. Tucker, ${ }^{143}$ P. Wittich, ${ }^{143}$ M. Zientek, ${ }^{143}$ S. Abdullin, ${ }^{144}$ M. Albrow, ${ }^{144}$ M. Alyari, ${ }^{144}$ G. Apollinari, ${ }^{144}$ A. Apresyan, ${ }^{144}$ A. Apyan, ${ }^{144}$

S. Banerjee, ${ }^{144}$ L. A. T. Bauerdick, ${ }^{144}$ A. Beretvas, ${ }^{144}$ J. Berryhill,${ }^{144}$ P. C. Bhat,${ }^{144}$ G. Bolla, ${ }^{144, a}$ K. Burkett, ${ }^{144}$

J. N. Butler ${ }^{144}$ A. Canepa,${ }^{144}$ G. B. Cerati,${ }^{144}$ H. W. K. Cheung, ${ }^{144}$ F. Chlebana, ${ }^{144}$ M. Cremonesi, ${ }^{144}$ J. Duarte, ${ }^{144}$ V. D. Elvira ${ }^{144}$ J. Freeman, ${ }^{144}$ Z. Gecse ${ }^{144}$ E. Gottschalk, ${ }^{144}$ L. Gray, ${ }^{144}$ D. Green, ${ }^{144}$ S. Grünendahl,${ }^{144}$ O. Gutsche, ${ }^{144}$ R. M. Harris, ${ }^{144}$ S. Hasegawa, ${ }^{144}$ J. Hirschauer, ${ }^{144}$ Z. Hu, ${ }^{144}$ B. Jayatilaka, ${ }^{144}$ S. Jindariani, ${ }^{144}$ M. Johnson, ${ }^{144}$ U. Joshi, ${ }^{144}$ B. Klima, ${ }^{144}$ B. Kreis, ${ }^{144}$ S. Lammel, ${ }^{144}$ D. Lincoln, ${ }^{144}$ R. Lipton, ${ }^{144}$ M. Liu, ${ }^{144}$ T. Liu, ${ }^{144}$ R. Lopes De Sá, ${ }^{144}$ J. Lykken, ${ }^{144}$ K. Maeshima, ${ }^{144}$ N. Magini, ${ }^{144}$ J. M. Marraffino, ${ }^{144}$ D. Mason, ${ }^{144}$ P. McBride, ${ }^{144}$ P. Merkel, ${ }^{144}$ S. Mrenna, ${ }^{144}$ S. Nahn, ${ }^{144}$ V. O’Dell, ${ }^{144}$ K. Pedro, ${ }^{144}$ O. Prokofyev, ${ }^{144}$ G. Rakness, ${ }^{144}$ L. Ristori, ${ }^{144}$ B. Schneider, ${ }^{144}$ E. Sexton-Kennedy, ${ }^{144}$ A. Soha, ${ }^{144}$ W. J. Spalding, ${ }^{144}$ L. Spiegel, ${ }^{144}$ S. Stoynev, ${ }^{144}$ J. Strait, ${ }^{144}$ N. Strobbe,${ }^{144}$ L. Taylor,${ }^{144}$ S. Tkaczyk, ${ }^{144}$ N. V. Tran, ${ }^{144}$ L. Uplegger, ${ }^{144}$ E. W. Vaandering, ${ }^{144}$ C. Vernieri, ${ }^{144}$ M. Verzocchi, ${ }^{144}$ R. Vidal,,${ }^{144}$ M. Wang, ${ }^{144}$ H. A. Weber, ${ }^{144}$ A. Whitbeck, ${ }^{144}$ D. Acosta, ${ }^{145}$ P. Avery, ${ }^{145}$ P. Bortignon, ${ }^{145}$ D. Bourilkov, ${ }^{145}$ A. Brinkerhoff, ${ }^{145}$ A. Carnes, ${ }^{145}$ M. Carver, ${ }^{145}$ D. Curry, ${ }^{145}$ R. D. Field, ${ }^{145}$ I. K. Furic, ${ }^{145}$ S. V. Gleyzer, ${ }^{145}$ B. M. Joshi, ${ }^{145}$ J. Konigsberg, ${ }^{145}$ A. Korytov, ${ }^{145}$ K. Kotov, ${ }^{145}$ P. Ma, ${ }^{145}$ K. Matchev, ${ }^{145}$ H. Mei, ${ }^{145}$ G. Mitselmakher, ${ }^{145}$ K. Shi, ${ }^{145}$ D. Sperka, ${ }^{145}$ N. Terentyev, ${ }^{145}$ L. Thomas, ${ }^{145}$ J. Wang, ${ }^{145}$ S. Wang, ${ }^{145}$ J. Yelton, ${ }^{145}$ Y. R. Joshi, ${ }^{146}$ S. Linn, ${ }^{146}$ P. Markowitz, ${ }^{146}$ J. L. Rodriguez, ${ }^{146}$ A. Ackert, ${ }^{147}$ T. Adams, ${ }^{147}$ A. Askew, ${ }^{147}$ S. Hagopian, ${ }^{147}$ V. Hagopian, ${ }^{147}$ K. F. Johnson, ${ }^{147}$ T. Kolberg, ${ }^{147}$ G. Martinez, ${ }^{147}$ T. Perry, ${ }^{147}$ H. Prosper, ${ }^{147}$ A. Saha, ${ }^{147}$ A. Santra, ${ }^{147}$ V. Sharma,${ }^{147}$ R. Yohay, ${ }^{147}$ M. M. Baarmand, ${ }^{148}$ V. Bhopatkar, ${ }^{148}$ S. Colafranceschi, ${ }^{148}$ M. Hohlmann, ${ }^{148}$ D. Noonan, ${ }^{148}$ T. Roy, ${ }^{148}$ F. Yumiceva, ${ }^{148}$ M. R. Adams, ${ }^{149}$ L. Apanasevich, ${ }^{149}$ D. Berry, ${ }^{149}$ R. R. Betts, ${ }^{149}$ R. Cavanaugh, ${ }^{149}$ X. Chen, ${ }^{149}$ O. Evdokimov, ${ }^{149}$ C. E. Gerber, ${ }^{149}$ D. A. Hangal,${ }^{149}$ D. J. Hofman, ${ }^{149}$ K. Jung, ${ }^{149}$ J. Kamin, ${ }^{149}$ I. D. Sandoval Gonzalez, ${ }^{149}$ M. B. Tonjes, ${ }^{149}$ H. Trauger, ${ }^{149}$ N. Varelas, ${ }^{149}$ H. Wang, ${ }^{149}$ Z. Wu, ${ }^{149}$ J. Zhang, ${ }^{149}$ B. Bilki, ${ }^{150, \mathrm{mmm}}$ W. Clarida, ${ }^{150}$ K. Dilsiz, ${ }^{150, \mathrm{nnn}}$ S. Durgut, ${ }^{150}$ R. P. Gandrajula, ${ }^{150}$ M. Haytmyradov, ${ }^{150}$ V. Khristenko, ${ }^{150}$ J.-P. Merlo, ${ }^{150}$ H. Mermerkaya, ${ }^{150,000}$ A. Mestvirishvili, ${ }^{150}$ A. Moeller, ${ }^{150}$ J. Nachtman, ${ }^{150}$ H. Ogul, ${ }^{150, p p p}$ Y. Onel, ${ }^{150}$ F. Ozok, ${ }^{150, \text { qqq }}$ A. Penzo, ${ }^{150}$ C. Snyder, ${ }^{150}$ E. Tiras, ${ }^{150}$ J. Wetzel,${ }^{150}$ K. Yi, ${ }^{150}$ B. Blumenfeld, ${ }^{151}$ A. Cocoros, ${ }^{151}$ N. Eminizer, ${ }^{151}$ D. Fehling, ${ }^{151}$ L. Feng, ${ }^{151}$ A. V. Gritsan, ${ }^{151}$ P. Maksimovic,${ }^{151}$ J. Roskes, ${ }^{151}$ U. Sarica, ${ }^{151}$ M. Swartz, ${ }^{151}$ M. Xiao, ${ }^{151}$ C. You, ${ }^{151}$ A. Al-bataineh, ${ }^{152}$ P. Baringer, ${ }^{152}$ A. Bean, ${ }^{152}$ S. Boren, ${ }^{152}$ J. Bowen, ${ }^{152}$ J. Castle, ${ }^{152}$ S. Khalil, ${ }^{152}$ A. Kropivnitskaya, ${ }^{152}$ D. Majumder, ${ }^{152}$ W. Mcbrayer, ${ }^{152}$ M. Murray, ${ }^{152}$ C. Rogan, ${ }^{152}$ C. Royon, ${ }^{152}$ S. Sanders, ${ }^{152}$ E. Schmitz ${ }^{152}$ J. D. Tapia Takaki, ${ }^{152}$ Q. Wang, ${ }^{152}$ A. Ivanov, ${ }^{153}$ K. Kaadze, ${ }^{153}$ Y. Maravin, ${ }^{153}$ A. Mohammadi, ${ }^{153}$ L. K. Saini, ${ }^{153}$ N. Skhirtladze, ${ }^{153}$ F. Rebassoo, ${ }^{154}$ D. Wright, ${ }^{154}$ A. Baden, ${ }^{155}$ O. Baron, ${ }^{155}$ A. Belloni, ${ }^{155}$ S. C. Eno, ${ }^{155}$ Y. Feng, ${ }^{155}$ C. Ferraioli, ${ }^{155}$ N. J. Hadley, ${ }^{155}$ S. Jabeen, ${ }^{155}$ G. Y. Jeng, ${ }^{155}$ R. G. Kellogg, ${ }^{155}$ J. Kunkle, ${ }^{155}$ A. C. Mignerey, ${ }^{155}$ F. Ricci-Tam, ${ }^{155}$ Y. H. Shin, ${ }^{155}$ A. Skuja, ${ }^{155}$ S. C. Tonwar, ${ }^{155}$ D. Abercrombie, ${ }^{156}$ B. Allen, ${ }^{156}$ V. Azzolini, ${ }^{156}$ R. Barbieri, ${ }^{156}$ 
A. Baty, ${ }^{156}$ R. Bi ${ }^{156}$ S. Brandt, ${ }^{156}$ W. Busza, ${ }^{156}$ I. A. Cali,${ }^{156}$ M. D’Alfonso, ${ }^{156}$ Z. Demiragli, ${ }^{156}$ G. Gomez Ceballos, ${ }^{156}$ M. Goncharov, ${ }^{156}$ D. Hsu, ${ }^{156}$ M. Hu, ${ }^{156}$ Y. Iiyama ${ }^{156}$ G. M. Innocenti, ${ }^{156}$ M. Klute, ${ }^{156}$ D. Kovalskyi, ${ }^{156}$ Y.-J. Lee, ${ }^{156}$

A. Levin, ${ }^{156}$ P. D. Luckey, ${ }^{156}$ B. Maier, ${ }^{156}$ A. C. Marini,,${ }^{156}$ C. Mcginn, ${ }^{156}$ C. Mironov, ${ }^{156}$ S. Narayanan, ${ }^{156}$ X. Niu, ${ }^{156}$

C. Paus, ${ }^{156}$ C. Roland, ${ }^{156}$ G. Roland, ${ }^{156}$ J. Salfeld-Nebgen, ${ }^{156}$ G. S. F. Stephans, ${ }^{156}$ K. Tatar, ${ }^{156}$ D. Velicanu, ${ }^{156}$ J. Wang, ${ }^{156}$

T. W. Wang, ${ }^{156}$ B. Wyslouch, ${ }^{156}$ A. C. Benvenuti, ${ }^{157}$ R. M. Chatterjee, ${ }^{157}$ A. Evans, ${ }^{157}$ P. Hansen, ${ }^{157}$ J. Hiltbrand, ${ }^{157}$

S. Kalafut, ${ }^{157}$ Y. Kubota, ${ }^{157}$ Z. Lesko, ${ }^{157}$ J. Mans ${ }^{157}$ S. Nourbakhsh,${ }^{157}$ N. Ruckstuhll ${ }^{157}$ R. Rusack, ${ }^{157}$ J. Turkewitz, ${ }^{157}$ M. A. Wadud, ${ }^{157}$ J. G. Acosta, ${ }^{158}$ S. Oliveros, ${ }^{158}$ E. Avdeeva, ${ }^{159}$ K. Bloom,${ }^{159}$ D. R. Claes, ${ }^{159}$ C. Fangmeier,${ }^{159}$ F. Golf, ${ }^{159}$ R. Gonzalez Suarez, ${ }^{159}$ R. Kamalieddin, ${ }^{159}$ I. Kravchenko, ${ }^{159}$ J. Monroy, ${ }^{159}$ J. E. Siado, ${ }^{159}$ G. R. Snow, ${ }^{159}$ B. Stieger, ${ }^{159}$ J. Dolen, ${ }^{160}$ A. Godshalk, ${ }^{160}$ C. Harrington, ${ }^{160}$ I. Iashvili, ${ }^{160}$ D. Nguyen, ${ }^{160}$ A. Parker, ${ }^{160}$ S. Rappoccio, ${ }^{160}$ B. Roozbahani, ${ }^{160}$ G. Alverson, ${ }^{161}$ E. Barberis, ${ }^{161}$ C. Freer ${ }^{161}$ A. Hortiangtham, ${ }^{161}$ A. Massironi, ${ }^{161}$ D. M. Morse ${ }^{161}$ T. Orimoto, ${ }^{161}$

R. Teixeira De Lima, ${ }^{161}$ D. Trocino, ${ }^{161}$ T. Wamorkar, ${ }^{161}$ B. Wang, ${ }^{161}$ A. Wisecarver, ${ }^{161} \mathrm{D}$. Wood, ${ }^{161} \mathrm{~S}$. Bhattacharya, ${ }^{162}$ O. Charaf, ${ }^{162}$ K. A. Hahn, ${ }^{162}$ N. Mucia, ${ }^{162}$ N. Odell, ${ }^{162}$ M. H. Schmitt, ${ }^{162}$ K. Sung, ${ }^{162}$ M. Trovato, ${ }^{162}$ M. Velasco, ${ }^{162}$

R. Bucci, ${ }^{163}$ N. Dev, ${ }^{163}$ M. Hildreth,${ }^{163}$ K. Hurtado Anampa, ${ }^{163}$ C. Jessop, ${ }^{163}$ D. J. Karmgard, ${ }^{163}$ N. Kellams, ${ }^{163}$

K. Lannon, ${ }^{163}$ W. Li, ${ }^{163}$ N. Loukas, ${ }^{163}$ N. Marinelli, ${ }^{163}$ F. Meng, ${ }^{163}$ C. Mueller, ${ }^{163}$ Y. Musienko, ${ }^{163,11}$ M. Planer, ${ }^{163}$

A. Reinsvold, ${ }^{163}$ R. Ruchti, ${ }^{163}$ P. Siddireddy, ${ }^{163}$ G. Smith, ${ }^{163}$ S. Taroni, ${ }^{163}$ M. Wayne, ${ }^{163}$ A. Wightman, ${ }^{163}$ M. Wolf, ${ }^{163}$ A. Woodard, ${ }^{163}$ J. Alimena, ${ }^{164}$ L. Antonelli, ${ }^{164}$ B. Bylsma, ${ }^{164}$ L. S. Durkin, ${ }^{164}$ S. Flowers, ${ }^{164}$ B. Francis, ${ }^{164}$ A. Hart, ${ }^{164}$ C. Hill, ${ }^{164}$ W. Ji, ${ }^{164}$ B. Liu, ${ }^{164}$ W. Luo, ${ }^{164}$ B. L. Winer, ${ }^{164}$ H. W. Wulsin, ${ }^{164}$ S. Cooperstein, ${ }^{165}$ O. Driga, ${ }^{165}$ P. Elmer, ${ }^{165}$ J. Hardenbrook, ${ }^{165}$ P. Hebda ${ }^{165}$ S. Higginbotham, ${ }^{165}$ A. Kalogeropoulos, ${ }^{165}$ D. Lange, ${ }^{165}$ J. Luo, ${ }^{165}$ D. Marlow, ${ }^{165}$ K. Mei, ${ }^{165}$ I. Ojalvo, ${ }^{165}$ J. Olsen, ${ }^{165}$ C. Palmer, ${ }^{165}$ P. Piroué, ${ }^{165}$ D. Stickland, ${ }^{165}$ C. Tully, ${ }^{165}$ S. Malik, ${ }^{166}$ S. Norberg, ${ }^{166}$ A. Barker, ${ }^{167}$ V.E. Barnes, ${ }^{167}$ S. Das, ${ }^{167}$ S. Folgueras, ${ }^{167}$ L. Gutay, ${ }^{167}$ M. Jones, ${ }^{167}$ A. W. Jung, ${ }^{167}$ A. Khatiwada, ${ }^{167}$ D. H. Miller, ${ }^{167}$ N. Neumeister, ${ }^{167}$ C. C. Peng, ${ }^{167}$ H. Qiu, ${ }^{167}$ J. F. Schulte, ${ }^{167}$ J. Sun, ${ }^{167}$ F. Wang ${ }^{167}$ R. Xiao, ${ }^{167}$ W. Xie, ${ }^{167}$ T. Cheng, ${ }^{168}$ N. Parashar, ${ }^{168}$ J. Stupak, ${ }^{168}$ Z. Chen, ${ }^{169}$ K. M. Ecklund, ${ }^{169}$ S. Freed, ${ }^{169}$ F. J. M. Geurts, ${ }^{169}$ M. Guilbaud, ${ }^{169}$ M. Kilpatrick, ${ }^{169}$ W. Li, ${ }^{169}$ B. Michlin, ${ }^{169}$ B. P. Padley, ${ }^{169}$ J. Roberts, ${ }^{169}$ J. Rorie, ${ }^{169}$ W. Shi, ${ }^{169}$ Z. Tu, ${ }^{169}$ J. Zabel, ${ }^{169}$ A. Zhang, ${ }^{169}$ A. Bodek, ${ }^{170}$ P. de Barbaro, ${ }^{170}$ R. Demina, ${ }^{170}$ Y. t. Duh, ${ }^{170}$ T. Ferbel, ${ }^{170}$ M. Galanti, ${ }^{170}$ A. Garcia-Bellido, ${ }^{170}$ J. Han,,${ }^{170}$ O. Hindrichs, ${ }^{170}$

A. Khukhunaishvili, ${ }^{170}$ K. H. Lo ${ }^{170}$ P. Tan, ${ }^{170}$ M. Verzetti, ${ }^{170}$ R. Ciesielski,${ }^{171}$ K. Goulianos, ${ }^{171}$ C. Mesropian,${ }^{171}$ A. Agapitos ${ }^{172}$ J. P. Chou, ${ }^{172}$ Y. Gershtein, ${ }^{172}$ T. A. Gómez Espinosa, ${ }^{172}$ E. Halkiadakis, ${ }^{172}$ M. Heindl,${ }^{172}$ E. Hughes,${ }^{172}$ S. Kaplan, ${ }^{172}$ R. Kunnawalkam Elayavalli, ${ }^{172}$ S. Kyriacou, ${ }^{172}$ A. Lath, ${ }^{172}$ R. Montalvo, ${ }^{172}$ K. Nash,${ }^{172}$ M. Osherson, ${ }^{172}$ H. Saka, ${ }^{172}$ S. Salur, ${ }^{172}$ S. Schnetzer, ${ }^{172}$ D. Sheffield, ${ }^{172}$ S. Somalwar, ${ }^{172}$ R. Stone, ${ }^{172}$ S. Thomas, ${ }^{172}$ P. Thomassen, ${ }^{172}$ M. Walker, ${ }^{172}$ A. G. Delannoy, ${ }^{173}$ J. Heideman, ${ }^{173}$ G. Riley, ${ }^{173}$ K. Rose, ${ }^{173}$ S. Spanier, ${ }^{173}$ K. Thapa, ${ }^{173}$ O. Bouhali, ${ }^{174, \text { rrr }}$ A. Castaneda Hernandez, ${ }^{174, \text { rrr }}$ A. Celik, ${ }^{174}$ M. Dalchenko, ${ }^{174}$ M. De Mattia, ${ }^{174}$ A. Delgado, ${ }^{174}$ S. Dildick, ${ }^{174}$ R. Eusebi, ${ }^{174}$ J. Gilmore, ${ }^{174}$ T. Huang, ${ }^{174}$ T. Kamon, ${ }^{174, \text { sss }}$ R. Mueller, ${ }^{174}$ Y. Pakhotin, ${ }^{174}$ R. Patel, ${ }^{174}$ A. Perloff,,${ }^{174}$ L. Perniè, ${ }^{174}$

D. Rathjens, ${ }^{174}$ A. Safonov, ${ }^{174}$ A. Tatarinov, ${ }^{174}$ K. A. Ulmer, ${ }^{174}$ N. Akchurin, ${ }^{175}$ J. Damgov, ${ }^{175}$ F. De Guio, ${ }^{175}$ P. R. Dudero, ${ }^{175}$ J. Faulkner, ${ }^{175}$ E. Gurpinar, ${ }^{175}$ S. Kunori, ${ }^{175}$ K. Lamichhane, ${ }^{175}$ S. W. Lee, ${ }^{175}$ T. Libeiro, ${ }^{175}$ T. Mengke, ${ }^{175}$ S. Muthumuni, ${ }^{175}$ T. Peltola, ${ }^{175}$ S. Undleeb, ${ }^{175}$ I. Volobouev, ${ }^{175}$ Z. Wang, ${ }^{175}$ S. Greene, ${ }^{176}$ A. Gurrola, ${ }^{176}$ R. Janjam, ${ }^{176}$ W. Johns,${ }^{176}$ C. Maguire, ${ }^{176}$ A. Melo, ${ }^{176}$ H. Ni, ${ }^{176}$ K. Padeken, ${ }^{176}$ P. Sheldon, ${ }^{176}$ S. Tuo, ${ }^{176}$ J. Velkovska, ${ }^{176}$ Q. Xu, ${ }^{176}$ M. W. Arenton, ${ }^{177}$ P. Barria, ${ }^{177}$ B. Cox,${ }^{177}$ R. Hirosky, ${ }^{177}$ M. Joyce, ${ }^{177}$ A. Ledovskoy, ${ }^{177}$ H. Li, ${ }^{177}$ C. Neu, ${ }^{177}$

T. Sinthuprasith, ${ }^{177}$ Y. Wang, ${ }^{177}$ E. Wolfe, ${ }^{177}$ F. Xia, ${ }^{177}$ R. Harr, ${ }^{178}$ P. E. Karchin, ${ }^{178}$ N. Poudyal, ${ }^{178}$ J. Sturdy,${ }^{178}$ P. Thapa, ${ }^{178}$ S. Zaleski, ${ }^{178}$ M. Brodski, ${ }^{179}$ J. Buchanan, ${ }^{179}$ C. Caillol, ${ }^{179}$ S. Dasu, ${ }^{179}$ L. Dodd,${ }^{179}$ S. Duric,${ }^{179}$ B. Gomber, ${ }^{179}$ M. Grothe,${ }^{179}$ M. Herndon, ${ }^{179}$ A. Hervé, ${ }^{179}$ U. Hussain, ${ }^{179}$ P. Klabbers, ${ }^{179}$ A. Lanaro, ${ }^{179}$ A. Levine, ${ }^{179}$ K. Long, ${ }^{179}$ R. Loveless, ${ }^{179}$ T. Ruggles, ${ }^{179}$ A. Savin, ${ }^{179}$ N. Smith, ${ }^{179}$ W. H. Smith, ${ }^{179}$ D. Taylor,,${ }^{179}$ and N. Woods ${ }^{179}$

(CMS Collaboration)

\footnotetext{
${ }^{1}$ Yerevan Physics Institute, Yerevan, Armenia

${ }^{2}$ Institut für Hochenergiephysik, Wien, Austria

${ }^{3}$ Institute for Nuclear Problems, Minsk, Belarus

${ }^{4}$ Universiteit Antwerpen, Antwerpen, Belgium

${ }^{5}$ Vrije Universiteit Brussel, Brussel, Belgium

${ }^{6}$ Université Libre de Bruxelles, Bruxelles, Belgium
} 


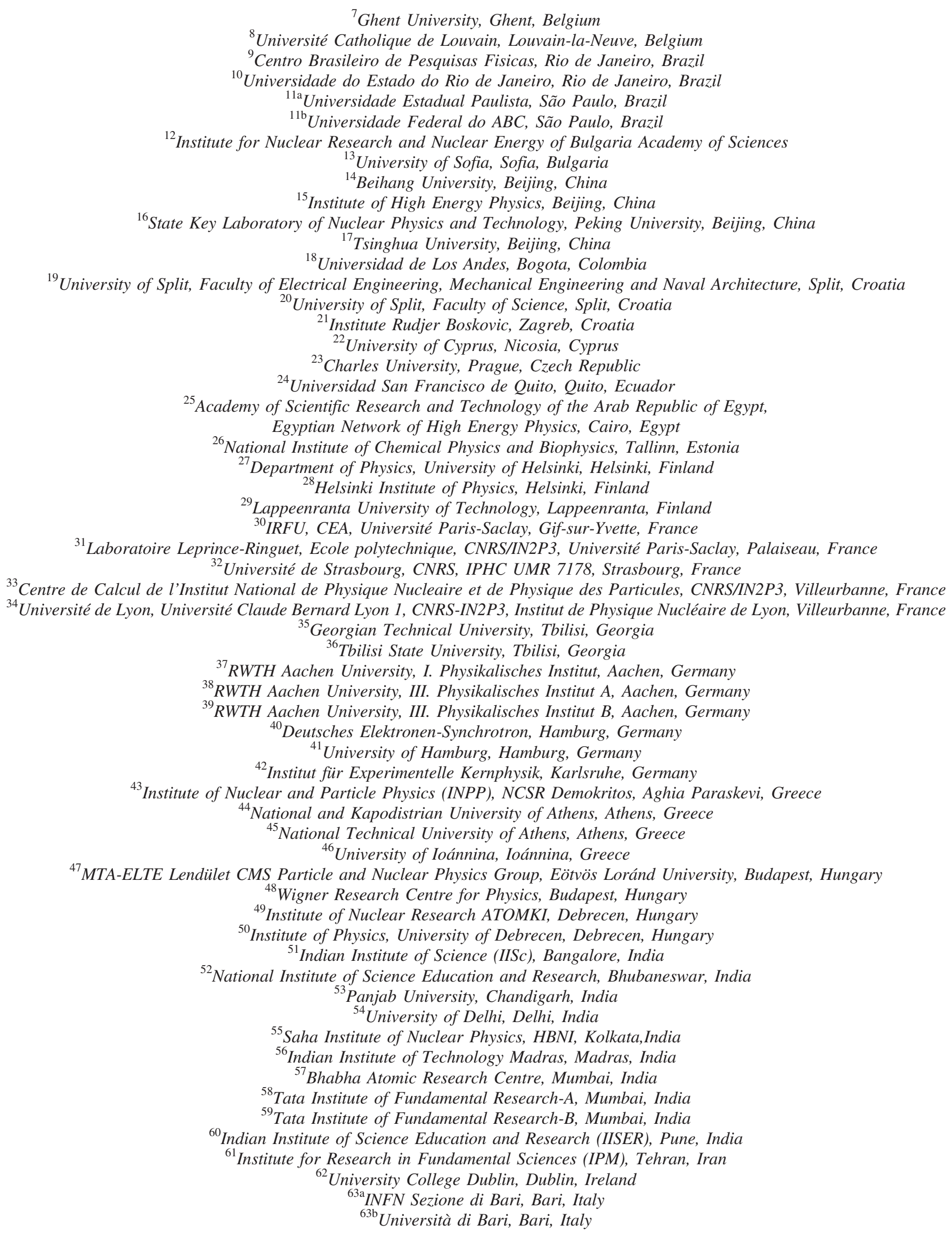


${ }^{63 \mathrm{c}}$ Politecnico di Bari, Bari, Italy

${ }^{64 \mathrm{a}}$ INFN Sezione di Bologna, Bologna, Italy

${ }^{64 \mathrm{~b}}$ Università di Bologna, Bologna, Italy

${ }^{65 a}$ INFN Sezione di Catania, Catania, Italy

${ }^{65 \mathrm{~b}}$ Università di Catania, Catania, Italy

${ }^{66 \mathrm{a}}$ INFN Sezione di Firenze, Firenze, Italy

${ }^{66 \mathrm{~b}}$ Università di Firenze, Firenze, Italy

${ }^{67}$ INFN Laboratori Nazionali di Frascati, Frascati, Italy

${ }^{68 a}$ INFN Sezione di Genova, Genova, Italy

${ }^{68 \mathrm{~b}}$ Università di Genova, Genova, Italy

${ }^{69 \mathrm{a}}$ INFN Sezione di Milano-Bicocca

${ }^{69 \mathrm{~b}}$ Università di Milano-Bicocca

${ }^{70 a}$ INFN Sezione di Napoli, Napoli, Italy

${ }^{70 \mathrm{~b}}$ Università di Napoli 'Federico II', Napoli, Italy

${ }^{70 \mathrm{c}}$ Università della Basilicata, Potenza, Italy

${ }^{70 \mathrm{~d}}$ Università G. Marconi, Roma, Italy

${ }^{71 a}$ INFN Sezione di Padova, Padova, Italy

${ }^{71 \mathrm{~b}}$ Università di Padova, Padova, Italy

${ }^{72 a}$ INFN Sezione di Pavia, Pavia, Italy

${ }^{72 \mathrm{~b}}$ Università di Pavia, Pavia, Italy

${ }^{73 a}$ INFN Sezione di Perugia, Perugia, Italy

${ }^{73 b}$ Università di Perugia, Perugia, Italy

${ }^{74 a}$ INFN Sezione di Pisa, Pisa, Italy

${ }^{74 b}$ Università di Pisa, Pisa, Italy

${ }^{74 \mathrm{c}}$ Scuola Normale Superiore di Pisa, Pisa, Italy

${ }^{75 a}$ INFN Sezione di Roma, Rome, Italy

${ }^{75 b}$ Sapienza Università di Roma, Rome, Italy

${ }^{76 a}$ INFN Sezione di Torino, Torino, Italy

${ }^{76 \mathrm{~b}}$ Università di Torino, Novara, Italy

${ }^{76 c}$ Università del Piemonte Orientale, Novara, Italy

${ }^{77 a}$ INFN Sezione di Trieste, Trieste, Italy

${ }^{77 \mathrm{~b}}$ Università di Trieste, Trieste, Italy

${ }^{78}$ Kyungpook National University, Daegu, Korea

${ }^{79}$ Chonbuk National University, Jeonju, Korea

${ }^{80}$ Chonnam National University, Institute for Universe and Elementary Particles, Kwangju, Korea

${ }^{81}$ Hanyang University, Seoul, Korea

${ }^{82}$ Korea University, Seoul, Korea

${ }^{83}$ Seoul National University, Seoul, Korea

${ }^{84}$ University of Seoul, Seoul, Korea

${ }^{85}$ Sungkyunkwan University, Suwon, Korea

${ }^{86}$ Vilnius University, Vilnius, Lithuania

${ }^{87}$ National Centre for Particle Physics, Universiti Malaya, Kuala Lumpur, Malaysia

${ }^{88}$ Centro de Investigacion y de Estudios Avanzados del IPN, Mexico City, Mexico

${ }^{89}$ Universidad Iberoamericana, Mexico City, Mexico

${ }^{90}$ Benemerita Universidad Autonoma de Puebla, Puebla, Mexico

${ }^{91}$ Universidad Autónoma de San Luis Potosí, San Luis Potosí, Mexico

${ }^{92}$ University of Auckland, Auckland, New Zealand

${ }^{93}$ University of Canterbury, Christchurch, New Zealand

${ }^{94}$ National Centre for Physics, Quaid-I-Azam University, Islamabad, Pakistan

${ }^{95}$ National Centre for Nuclear Research, Swierk, Poland

${ }^{96}$ Institute of Experimental Physics, Faculty of Physics, University of Warsaw, Warsaw, Poland

${ }^{97}$ Laboratório de Instrumentação e Física Experimental de Partículas, Lisboa, Portugal

${ }^{98}$ Joint Institute for Nuclear Research, Dubna, Russia

${ }^{99}$ Petersburg Nuclear Physics Institute, Gatchina (St. Petersburg), Russia

${ }^{100}$ Institute for Nuclear Research, Moscow, Russia

${ }^{101}$ Institute for Theoretical and Experimental Physics, Moscow, Russia

${ }^{102}$ Moscow Institute of Physics and Technology, Moscow, Russia

${ }^{103}$ National Research Nuclear University 'Moscow Engineering Physics Institute' (MEPhI), Moscow, Russia

${ }^{104}$ P.N. Lebedev Physical Institute, Moscow, Russia

${ }^{105}$ Skobeltsyn Institute of Nuclear Physics, Lomonosov Moscow State University, Moscow, Russia 
${ }^{106}$ Novosibirsk State University (NSU), Novosibirsk, Russia

${ }^{107}$ State Research Center of Russian Federation, Institute for High Energy Physics, Protvino, Russia

${ }^{108}$ University of Belgrade, Faculty of Physics and Vinca Institute of Nuclear Sciences, Belgrade, Serbia

${ }^{109}$ Centro de Investigaciones Energéticas Medioambientales y Tecnológicas (CIEMAT), Madrid, Spain

${ }^{110}$ Universidad Autónoma de Madrid, Madrid, Spain

${ }^{111}$ Universidad de Oviedo, Oviedo, Spain

${ }^{112}$ Instituto de Física de Cantabria (IFCA), CSIC-Universidad de Cantabria, Santander, Spain

${ }^{113}$ CERN, European Organization for Nuclear Research, Geneva, Switzerland

${ }^{114}$ Paul Scherrer Institut, Villigen, Switzerland

${ }^{115}$ Institute for Particle Physics and Astrophysics (IPA), Zurich, Switzerland

${ }^{116}$ Universität Zürich, Zurich, Switzerland

${ }^{117}$ National Central University, Chung-Li, Taiwan

${ }^{118}$ National Taiwan University (NTU), Taipei, Taiwan

${ }^{119}$ Chulalongkorn University, Faculty of Science, Department of Physics, Bangkok, Thailand

${ }^{120}$ Çukurova University, Physics Department, Science and Art Faculty, Adana, Turkey

${ }^{121}$ Middle East Technical University, Physics Department, Ankara, Turkey

${ }^{122}$ Bogazici University, Istanbul, Turkey

${ }^{123}$ Istanbul Technical University, Istanbul, Turkey

${ }^{124}$ Institute for Scintillation Materials of National Academy of Science of Ukraine, Kharkov, Ukraine

${ }^{125}$ National Scientific Center, Kharkov Institute of Physics and Technology, Kharkov, Ukraine

${ }^{126}$ University of Bristol, Bristol, United Kingdom

${ }^{127}$ Rutherford Appleton Laboratory, Didcot, United Kingdom

${ }^{128}$ Imperial College, London, United Kingdom

${ }^{129}$ Brunel University, Uxbridge, United Kingdom

${ }^{130}$ Baylor University, Waco, Texas, USA

${ }^{131}$ Catholic University of America, Washington DC, USA

${ }^{132}$ The University of Alabama, Tuscaloosa, Alabama, USA

${ }^{133}$ Boston University, Boston, Massachusetts, USA

${ }^{134}$ Brown University, Providence, Rhode Island, USA

${ }^{135}$ University of California, Davis, Davis, California, USA

${ }^{136}$ University of California, Los Angeles, California, USA

${ }^{137}$ University of California, Riverside, Riverside, California, USA

${ }^{138}$ University of California, San Diego, La Jolla, California, USA

${ }^{139}$ University of California, Santa Barbara - Department of Physics, Santa Barbara, California, USA

${ }^{140}$ California Institute of Technology, Pasadena, California, USA

${ }^{141}$ Carnegie Mellon University, Pittsburgh, Pennsylvania, USA

${ }^{142}$ University of Colorado Boulder, Boulder, Colorado, USA

${ }^{143}$ Cornell University, Ithaca, New York, USA

${ }^{144}$ Fermi National Accelerator Laboratory, Batavia, Illinois, USA

${ }^{145}$ University of Florida, Gainesville, Florida, USA

${ }^{146}$ Florida International University, Miami, Florida, USA

${ }^{147}$ Florida State University, Tallahassee, Florida, USA

${ }^{148}$ Florida Institute of Technology, Melbourne, Florida, USA

${ }^{149}$ University of Illinois at Chicago (UIC), Chicago, Illinois, USA

${ }^{150}$ The University of Iowa, Iowa City, Iowa, USA

${ }^{151}$ Johns Hopkins University, Baltimore, Maryland, USA

${ }^{152}$ The University of Kansas, Lawrence, Kansas, USA

${ }^{153}$ Kansas State University, Manhattan, Kansas, USA

${ }^{154}$ Lawrence Livermore National Laboratory, Livermore, California, USA

${ }^{155}$ University of Maryland, College Park, Maryland, USA

${ }^{156}$ Massachusetts Institute of Technology, Cambridge, Massachusetts, USA

${ }^{157}$ University of Minnesota, Minneapolis, Minnesota, USA

${ }^{158}$ University of Mississippi, Oxford, Mississippi, USA

${ }^{159}$ University of Nebraska-Lincoln, Lincoln, Nebraska, USA

${ }^{160}$ State University of New York at Buffalo, Buffalo, New York, USA

${ }^{161}$ Northeastern University, Boston, Massachusetts, USA

${ }^{162}$ Northwestern University, Evanston, Illinois, USA

${ }^{163}$ University of Notre Dame, Notre Dame, Indiana, USA

${ }^{164}$ The Ohio State University, Columbus, Ohio, USA

${ }^{165}$ Princeton University, Princeton, New Jersey, USA 


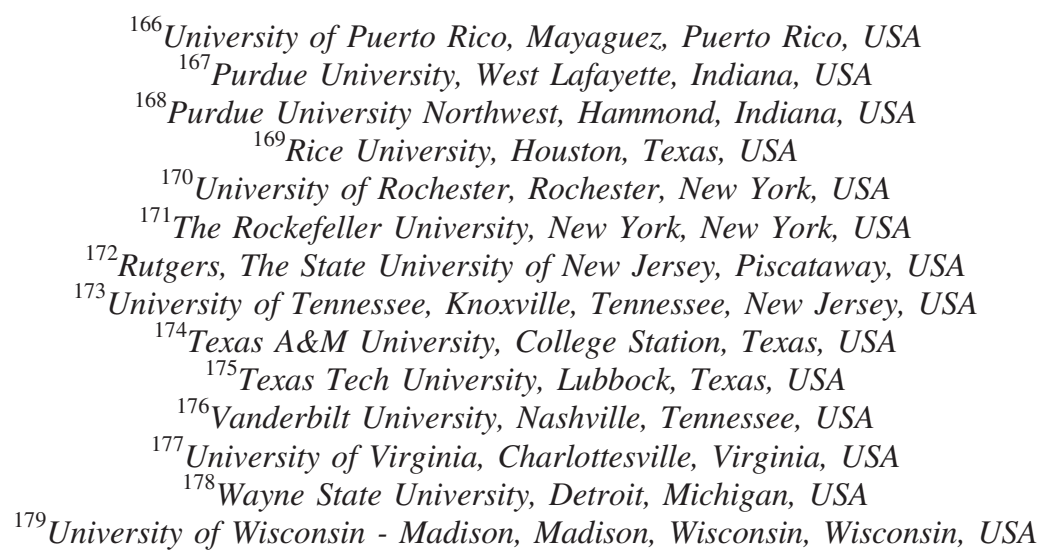

${ }^{\mathrm{a}}$ Deceased.

${ }^{\mathrm{b}}$ Also at Vienna University of Technology, Vienna, Austria.

${ }^{c}$ Also at IRFU, CEA, Université Paris-Saclay, Gif-sur-Yvette, France.

${ }^{\mathrm{d}}$ Also at Universidade Estadual de Campinas, Campinas, Brazil.

${ }^{\mathrm{e}}$ Also at Universidade Federal de Pelotas, Pelotas, Brazil.

${ }^{\mathrm{f}}$ Also at Université Libre de Bruxelles, Bruxelles, Belgium.

${ }^{g}$ Also at Institute for Theoretical and Experimental Physics, Moscow, Russia.

${ }^{\mathrm{h}}$ Also at Joint Institute for Nuclear Research, Dubna, Russia.

${ }^{\mathrm{i}}$ Also at Suez University, Suez, Egypt.

${ }^{\mathrm{j}}$ Also at British University in Egypt, Cairo, Egypt.

${ }^{\mathrm{k}}$ Also at Helwan University, Cairo, Egypt.

${ }^{1}$ Also at King Abdulaziz University, Jeddah, Saudi Arabia, Jeddah, Saudi Arabia.

${ }^{\mathrm{m}}$ Also at Université de Haute Alsace, Mulhouse, France.

${ }^{\mathrm{n}}$ Also at Skobeltsyn Institute of Nuclear Physics, Lomonosov Moscow State University, Moscow, Russia.

${ }^{\circ}$ Also at Tbilisi State University, Tbilisi, Georgia.

${ }^{\mathrm{p}}$ Also at Ilia State University, Tbilisi, Georgia.

${ }^{\mathrm{q}}$ Also at CERN, European Organization for Nuclear Research, Geneva, Switzerland.

${ }^{\mathrm{r}}$ Also at RWTH Aachen University, III. Physikalisches Institut A, Aachen, Germany.

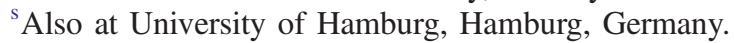

${ }^{\mathrm{t}}$ Also at Brandenburg University of Technology, Cottbus, Germany.

"Also at MTA-ELTE Lendület CMS Particle and Nuclear Physics Group, Eötvös Loránd University, Budapest, Hungary.

${ }^{v}$ Also at Institute of Nuclear Research ATOMKI, Debrecen, Hungary.

${ }^{\mathrm{w}}$ Also at Institute of Physics, University of Debrecen, Debrecen, Hungary.

${ }^{\mathrm{x}}$ Also at IIT Bhubaneswar, Bhubaneswar, India.

${ }^{\mathrm{y}}$ Also at Institute of Physics, Bhubaneswar, India.

${ }^{\mathrm{z}}$ Also at University of Visva-Bharati, Santiniketan, India.

${ }^{\text {aa }}$ Also at University of Ruhuna, Matara, Sri Lanka.

${ }^{\mathrm{bb}}$ Also at Isfahan University of Technology, Isfahan, Iran.

${ }^{c c}$ Also at Yazd University, Yazd, Iran.

${ }^{\mathrm{dd}}$ Also at Plasma Physics Research Center, Science and Research Branch, Islamic Azad University, Tehran, Iran.

${ }^{e e}$ Also at Università degli Studi di Siena, Siena, Italy.

${ }^{\mathrm{ff}}$ Also at INFN Sezione di Milano-Bicocca, Università di Milano-Bicocca, Milano, Italy.

${ }^{\mathrm{gg}}$ Also at Purdue University, West Lafayette, USA.

${ }^{\mathrm{hh}}$ Also at International Islamic University of Malaysia, Kuala Lumpur, Malaysia.

${ }^{\text {ii } A l s o ~ a t ~ M a l a y s i a n ~ N u c l e a r ~ A g e n c y, ~ M O S T I, ~ K a j a n g, ~ M a l a y s i a . ~}$

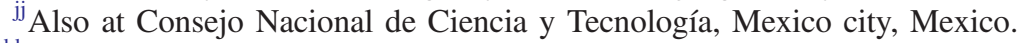

${ }^{\mathrm{kk}}$ Also at Warsaw University of Technology, Institute of Electronic Systems, Warsaw, Poland.

${ }^{11}$ Also at Institute for Nuclear Research, Moscow, Russia.

${ }^{\mathrm{mm}}$ Also at National Research Nuclear University 'Moscow Engineering Physics Institute' (MEPhI), Moscow, Russia.

${ }^{n n}$ Also at St. Petersburg State Polytechnical University, St. Petersburg, Russia.

${ }^{\text {oo }}$ Also at University of Florida, Gainesville, USA.

${ }^{\mathrm{pp}}$ Also at P.N. Lebedev Physical Institute, Moscow, Russia.

${ }^{\mathrm{qq}}$ Also at Budker Institute of Nuclear Physics, Novosibirsk, Russia.

${ }^{\mathrm{rr}}$ Also at Faculty of Physics, University of Belgrade, Belgrade, Serbia.

${ }^{\text {ss }}$ Also at University of Belgrade, Faculty of Physics and Vinca Institute of Nuclear Sciences, Belgrade, Serbia. 
${ }^{\text {tt} A l s o ~ a t ~ S c u o l a ~ N o r m a l e ~ e ~ S e z i o n e ~ d e l l ' I N F N, ~ P i s a, ~ I t a l y . ~}$

${ }^{\mathrm{uu}}$ Also at National and Kapodistrian University of Athens, Athens, Greece.

${ }^{\mathrm{vv}}$ Also at Riga Technical University, Riga, Latvia.

${ }^{\text {ww }}$ Also at Universität Zürich, Zurich, Switzerland.

${ }^{\mathrm{xx}}$ Also at Stefan Meyer Institute for Subatomic Physics.

${ }^{\text {yy }}$ Also at Adiyaman University, Adiyaman, Turkey.

${ }^{\mathrm{zz}}$ Also at Istanbul Aydin University, Istanbul, Turkey.

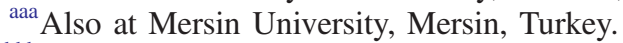

${ }^{b b b}$ Also at Cag University, Mersin, Turkey.

${ }^{\mathrm{ccc}}$ Also at Piri Reis University, Istanbul, Turkey.

${ }^{\mathrm{ddd}}$ Also at Izmir Institute of Technology, Izmir, Turkey.

${ }^{e e e}$ Also at Necmettin Erbakan University, Konya, Turkey.

${ }^{\mathrm{fff}}$ Also at Marmara University, Istanbul, Turkey.

${ }^{\text {ggg }}$ Also at Kafkas University, Kars, Turkey.

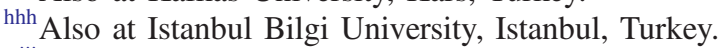

iii Also at Rutherford Appleton Laboratory, Didcot, United Kingdom.

${ }^{j j j}$ Also at School of Physics and Astronomy, University of Southampton, Southampton, United Kingdom.

${ }^{k k k}$ Also at Instituto de Astrofísica de Canarias, La Laguna, Spain.

${ }^{111}$ Also at Utah Valley University, Orem, USA.

${ }^{\mathrm{mmm}}$ Also at Beykent University.

${ }^{n n n}$ Also at Bingol University, Bingol, Turkey.

${ }^{\circ 00}$ Also at Erzincan University, Erzincan, Turkey.

${ }^{\mathrm{ppp}}$ Also at Sinop University, Sinop, Turkey.

${ }^{\mathrm{qqq}} \mathrm{Also}$ at Mimar Sinan University, Istanbul, Istanbul, Turkey.

${ }^{\mathrm{rrr}}$ Also at Texas A\&M University at Qatar, Doha, Qatar.

${ }^{\text {sss }}$ Also at Kyungpook National University, Daegu, Korea. 